\title{
Equality of averaged and quenched large deviations for random walks in random environments in dimensions four and higher
}

\author{
Atilla Yilmaz
}

Received: 9 March 2009 / Revised: 31 December 2009 / Published online: 5 February 2010

(C) The Author(s) 2010. This article is published with open access at Springerlink.com

\begin{abstract}
We consider large deviations for nearest-neighbor random walk in a uniformly elliptic i.i.d. environment. It is easy to see that the quenched and the averaged rate functions are not identically equal. When the dimension is at least four and Sznitman's transience condition (T) is satisfied, we prove that these rate functions are finite and equal on a closed set whose interior contains every nonzero velocity at which the rate functions vanish.
\end{abstract}

Keywords Large deviations - Random walk · Random environment - Disordered media $\cdot$ Renewal theorem

Mathematics Subject Classification (2000) $\quad 60 \mathrm{~K} 37 \cdot 60 \mathrm{~F} 10 \cdot 82 \mathrm{C} 41$

\section{Introduction}

\subsection{The model}

Let $U:=\left\{ \pm e_{i}\right\}_{i=1}^{d}$ where $\left(e_{i}\right)_{i=1}^{d}$ denotes the canonical basis for the $d$-dimensional integer lattice $\mathbb{Z}^{d}$ with $d \geq 1$. Consider a discrete time Markov chain on $\mathbb{Z}^{d}$ with nearest-neighbor steps, i.e., with steps in $U$. For every $x \in \mathbb{Z}^{d}$ and $z \in U$, the transition probability from $x$ to $x+z$ is denoted by $\pi(x, x+z)$, and the transition vector

\footnotetext{
A. Yilmaz

Faculty of Mathematics, Weizmann Institute of Science, 76100 Rehovot, Israel

Present Address:

A. Yilmaz $(\bowtie)$

Department of Mathematics, University of California, Berkeley, CA 94720-3840, USA

e-mail: atilla@math.berkeley.edu
} 
$\omega_{x}:=(\pi(x, x+z))_{z \in U}$ is referred to as the environment at $x$. If the environment $\omega:=\left(\omega_{x}\right)_{x \in \mathbb{Z}^{d}}$ is sampled from a probability space $(\Omega, \mathcal{B}, \mathbb{P})$, then this process is called random walk in a random environment (RWRE). Here, $\mathcal{B}$ is the Borel $\sigma$-algebra corresponding to the product topology.

For every $y \in \mathbb{Z}^{d}$, define the shift $T_{y}$ on $\Omega$ by $\left(T_{y} \omega\right)_{x}:=\omega_{x+y}$. Assume that $\mathbb{P}$ is stationary and ergodic under $\left(T_{z}\right)_{z \in U}$ and

$$
\begin{aligned}
& \text { there exists a } \delta>0 \text { such that } \mathbb{P}\{\pi(0, z) \geq \delta\}=1 \\
& \text { for every } z \in U \text {. (Uniform ellipticity.) }
\end{aligned}
$$

For every $x \in \mathbb{Z}^{d}$ and $\omega \in \Omega$, the Markov chain with environment $\omega$ induces a probability measure $P_{x}^{\omega}$ on the space of paths starting at $x$. Statements about $P_{x}^{\omega}$ that hold for $\mathbb{P}$-a.e. $\omega$ are referred to as quenched. Statements about the semi-direct product $P_{x}:=\mathbb{P} \times P_{x}^{\omega}$ are referred to as averaged (or annealed). Expectations under $\mathbb{P}, P_{x}^{\omega}$ and $P_{x}$ are denoted by $\mathbb{E}, E_{x}^{\omega}$ and $E_{x}$, respectively.

See [24] for a survey of results on RWRE.

\subsection{Regeneration times}

Let $\left(X_{n}\right)_{n \geq 0}$ denote the path of a particle taking a RWRE. Consider a unit vector $\hat{u} \in \mathcal{S}^{d-1}$. Define a sequence $\left(\tau_{m}\right)_{m \geq 0}=\left(\tau_{m}(\hat{u})\right)_{m \geq 0}$ of random times, which are referred to as regeneration times (relative to $\hat{u}$ ), by $\tau_{o}:=0$ and

$$
\tau_{m}:=\inf \left\{j>\tau_{m-1}:\left\langle X_{i}, \hat{u}\right\rangle<\left\langle X_{j}, \hat{u}\right\rangle \leq\left\langle X_{k}, \hat{u}\right\rangle \text { for all } i, k \text { with } i<j<k\right\}
$$

for every $m \geq 1$. If the walk is directionally transient relative to $\hat{u}$, i.e., if

$$
P_{o}\left(\lim _{n \rightarrow \infty}\left\langle X_{n}, \hat{u}\right\rangle=\infty\right)=1
$$

then $P_{o}\left(\tau_{m}<\infty\right)=1$ for every $m \geq 1$. As noted in [19], the significance of $\left(\tau_{m}\right)_{m \geq 1}$ is due to the fact that

$$
\left(X_{\tau_{m}+1}-X_{\tau_{m}}, X_{\tau_{m}+2}-X_{\tau_{m}}, \ldots, X_{\tau_{m+1}}-X_{\tau_{m}}\right)_{m \geq 1}
$$

is an i.i.d. sequence under $P_{o}$ when

$$
\omega=\left(\omega_{x}\right)_{x \in \mathbb{Z}^{d}} \text { is an i.i.d. collection. }
$$

The walk is said to satisfy Sznitman's transience condition $(\mathbf{T}, \hat{u})$ if (1.3) holds and

$$
E_{o}\left[\sup _{1 \leq i \leq \tau_{1}(\hat{u})} \exp \left\{\kappa_{1}\left|X_{i}\right|\right\}\right]<\infty \quad \text { for some } \kappa_{1}>0 .
$$


When $d \geq 2$, Sznitman [18] proves that (1.1), (1.4) and (T, $\hat{u})$ imply a ballistic law of large numbers (LLN), an averaged central limit theorem and certain large deviation estimates. Denote the LLN velocity by $\xi_{o} \neq 0$.

As stated below in Lemma $7,(\mathbf{T}, \hat{u})$ is satisfied as soon as the walk is non-nestling relative to $\hat{u}$, i.e., when

$$
\operatorname{ess} \inf _{\mathbb{P}} \sum_{z \in U} \pi(0, z)\langle z, \hat{u}\rangle>0
$$

The walk is said to be non-nestling if it is non-nestling relative to some unit vector. Otherwise, it is referred to as nestling. In the latter case, the convex hull of the support of the law of $\sum_{z} \pi(0, z) z$ contains the origin.

\subsection{Previous results on large deviations for RWRE}

Recall that a sequence $\left(Q_{n}\right)_{n \geq 1}$ of probability measures on a topological space $\mathbb{X}$ satisfies the large deviation principle (LDP) with rate function $I: \mathbb{X} \rightarrow \mathbb{R}$ if $I$ is non-negative, lower semicontinuous, and for any measurable set $G$,

$$
-\inf _{x \in G^{o}} I(x) \leq \liminf _{n \rightarrow \infty} \frac{1}{n} \log Q_{n}(G) \leq \limsup _{n \rightarrow \infty} \frac{1}{n} \log Q_{n}(G) \leq-\inf _{x \in \bar{G}} I(x) .
$$

Here, $G^{o}$ is the interior of $G$, and $\bar{G}$ its closure. See [8] for general background regarding large deviations.

Theorem 1 (Quenched LDP) For $\mathbb{P}$-a.e. $\omega,\left(P_{o}^{\omega}\left(\frac{X_{n}}{n} \in \cdot\right)\right)_{n \geq 1}$ satisfies the LDP with a deterministic and convex rate function $I_{q}$.

When $d=1$, Greven and den Hollander [10] prove Theorem 1 for walks in i.i.d. environments. They provide a formula for $I_{q}$ and show that its graph typically has flat pieces. Comets et al. [4] generalize the results in [10] to stationary and ergodic environments.

When $d \geq 1$, Zerner [25] proves Theorem 1 for nestling walks in i.i.d. environments. Varadhan [20] drops the nestling assumption and generalizes Theorem 1 to stationary and ergodic environments. Since both of these works rely on the subadditive ergodic theorem, they do not lead to any formulae for the rate function. Rosenbluth [16] gives an alternative proof of Theorem 1 in the case of stationary and ergodic environments. He provides a variational formula for the rate function $I_{q}$. In [23], we prove a quenched LDP for the pair empirical measure of the so-called environment Markov chain $\left(T_{X_{n}} \omega\right)_{n \geq 0}$. This implies Rosenbluth's result by an appropriate contraction.

In their aforementioned paper concerning RWRE on $\mathbb{Z}$, Comets et al. [4] prove also

Theorem 2 (Averaged LDP) $\left(P_{o}\left(\frac{X_{n}}{n} \in \cdot\right)\right)_{n \geq 1}$ satisfies the LDP with a convex rate function $I_{a}$. 
They establish this result for a class of environments including the i.i.d. case, and obtain the following variational formula for $I_{a}$ :

$$
I_{a}(\xi)=\inf _{\mathbb{Q}}\left\{I_{q}^{\mathbb{Q}}(\xi)+|\xi| h(\mathbb{Q} \mid \mathbb{P})\right\}
$$

Here, the infimum is over all stationary and ergodic probability measures on $\Omega, I_{q}^{\mathbb{Q}}(\cdot)$ denotes the rate function for the quenched LDP when the environment measure is $\mathbb{Q}$, and $h(\cdot \mid \cdot)$ is specific relative entropy. Similar to the quenched picture, the graph of $I_{a}$ is shown to typically have flat pieces.

Varadhan [20] considers walks in i.i.d. environments, and proves Theorem 2 for any $d \geq 1$. He gives a variational formula for $I_{a}$. (His formula does not resemble (1.7) in any way.) Rassoul-Agha [13] generalizes Varadhan's result to a class of mixing environments.

Let $\mathcal{N}_{q}:=\left\{\xi \in \mathbb{R}^{d}: I_{q}(\xi)=0\right\}$ and $\mathcal{N}_{a}:=\left\{\xi \in \mathbb{R}^{d}: I_{a}(\xi)=0\right\}$ denote the zero-sets of $I_{q}$ and $I_{a}$. The following theorem summarizes the previous results regarding the qualitative properties of the quenched and the averaged rate functions when $d \geq 2$.

Theorem 3 Assume $d \geq 2$, (1.1) and (1.4).

(a) $I_{q}$ and $I_{a}$ are convex, $I_{q}(0)=I_{a}(0)$ and $\mathcal{N}_{q}=\mathcal{N}_{a}$, cf. [20].

(b) If the walk is non-nestling, then

(i) $\mathcal{N}_{a}$ consists of the true velocity $\xi_{o}$, cf. [20], and

(ii) $I_{a}$ is strictly convex and analytic on an open set $\mathcal{A}_{a}$ containing $\xi_{o}$, cf. [12,21].

(c) If the walk is nestling, then $\mathcal{N}_{a}$ is a line segment containing the origin, $c f$. [20].

(d) If $(\boldsymbol{T}, \hat{u})$ is satisfied for some $\hat{u} \in \mathcal{S}^{d-1}$ in the latter case, then

(i) the origin is an endpoint of $\mathcal{N}_{a}$, cf. [18],

(ii) $I_{a}$ is strictly convex and analytic on an open set $\mathcal{A}_{a}^{+}, c f$. [21],

(iii) there exists a $(d-1)$-dimensional smooth surface patch $\mathcal{A}_{a}^{b}$ such that $\xi_{o} \in$ $\mathcal{A}_{a}^{b} \subset \partial \mathcal{A}_{a}^{+}, c f .[21]$

(iv) the unit vector $\eta_{o}$ normal to $\mathcal{A}_{a}^{b}$ (and pointing in $\mathcal{A}_{a}^{+}$) at $\xi_{o}$ satisfies $\left\langle\eta_{o}, \xi_{o}\right\rangle>$ $0, c f$. [21], and

(v) $I_{a}(t \xi)=t I_{a}(\xi)$ for every $\xi \in \mathcal{A}_{a}^{b}$ and $t \in[0,1]$, cf. [12].

\subsection{The main result}

Assume (1.1) and (1.4). It is clear that

$$
\begin{aligned}
\mathcal{D} & :=\left\{\left(\xi_{1}, \ldots, \xi_{d}\right) \in \mathbb{R}^{d}:\left|\xi_{1}\right|+\cdots+\left|\xi_{d}\right| \leq 1\right\}=\left\{\xi \in \mathbb{R}^{d}: I_{a}(\xi)<\infty\right\} \\
& =\left\{\xi \in \mathbb{R}^{d}: I_{q}(\xi) \leq-\log \delta\right\} .
\end{aligned}
$$

For any $\xi \in \mathbb{R}^{d}, I_{a}(\xi) \leq I_{q}(\xi)$ by Jensen's inequality and Fatou's lemma.

Proposition 4 If the support of $\mathbb{P}$ is not a singleton, then $I_{a}<I_{q}$ at some interior points of $\mathcal{D}$. 
Proof If the support of $\mathbb{P}$ is not a singleton, then $\mathbb{P}\{\pi(0, z)=\mathbb{E}\{\pi(0, z)\}\}<1$ for some $z \in U$, and

$$
\mathbb{E}\{\log \pi(0, z)\}<\log \mathbb{E}\{\pi(0, z)\}
$$

by Jensen's inequality. For every $n \geq 1$, the event $\left\{X_{n}=n z\right\}$ consists of a single path marching in the $z$-direction. In particular, this path never visits the same point more than once. Therefore,

$$
\log \mathbb{E}\{\pi(0, z)\}=\lim _{n \rightarrow \infty} \frac{1}{n} \log P_{o}\left(X_{n}=n z\right) \leq-I_{a}(z) .
$$

On the other hand, for every $\epsilon>0$,

$$
-I_{q}(z) \leq \liminf _{n \rightarrow \infty} \frac{1}{n} \log P_{o}^{\omega}\left(\left\langle X_{n}, z\right\rangle>n(1-\epsilon)\right) \leq(1-\epsilon) \mathbb{E}\{\log \pi(0, z)\}+O(\epsilon) .
$$

Explanation: For every $n \geq 1$, the number of paths constituting the event $\left\{\left\langle X_{n}, z\right\rangle\right\rangle$ $n(1-\epsilon)\}$ is $\mathrm{e}^{n O(\epsilon)}$. The probability of each such path is bounded from above by the product of the probabilities of its jumps in the $z$-direction taking place at distinct points. Since there are at least $n(1-\epsilon)$ such jumps, (1.10) follows from Jensen's inequality and the LLN for i.i.d. random variables.

Putting (1.8), (1.9) and (1.10) together, we conclude that $I_{a}(z)<I_{q}(z)$. Since the rate functions are convex and lower semicontinuous, they are in fact continuous on $\mathcal{D}$, cf. Theorem 10.2 of [15]. This implies the desired result.

The following theorem is the main result of this paper.

Theorem 5 Assume $d \geq 4$, (1.1), (1.4) and $(\boldsymbol{T}, \hat{u})$ for some $\hat{u} \in \mathcal{S}^{d-1}$.

(a) If the walk is non-nestling, then $I_{q}=I_{a}$ on an open set $\mathcal{A}_{e q}$ containing $\xi_{o}$.

(b) If the walk is nestling, then

(i) $I_{q}=I_{a}$ on an open set $\mathcal{A}_{e q}^{+}$,

(ii) there exists a $(d-1)$-dimensional smooth surface patch $\mathcal{A}_{\text {eq }}^{b}$ such that $\xi_{o} \in$ $\mathcal{A}_{e q}^{b} \subset \partial \mathcal{A}_{e q}^{+}$,

(iii) the unit vector $\eta_{o}$ normal to $\mathcal{A}_{e q}^{b}$ (and pointing in $\mathcal{A}_{\text {eq }}^{+}$) at $\xi_{o}$ satisfies $\left\langle\eta_{o}, \xi_{o}\right\rangle>0$, and

(iv) $I_{q}(t \xi)=t I_{q}(\xi)=t I_{a}(\xi)=I_{a}(t \xi)$ for every $\xi \in \mathcal{A}_{\text {eq }}^{b}$ and $t \in[0,1]$.

\section{Some remarks}

1. Since $I_{q}$ and $I_{a}$ are both continuous on $\mathcal{D}$, it is clear that $\mathcal{E}:=\left\{\xi \in \mathcal{D}: I_{q}(\xi)=\right.$ $\left.I_{a}(\xi)\right\}$ is closed. Proposition 4 and Theorem 5 imply that $\mathcal{D} \backslash \mathcal{E}$ and $\mathcal{E}$ both have nonempty interiors.

2. Assuming $d=1,(1.1)$ and (1.4), Comets et al. [4] use (1.7) to show that $I_{q}(\xi)=$ $I_{a}(\xi)$ if and only if $\xi=0$ or $I_{a}(\xi)=0$. In particular, Theorem 5 cannot be generalized to $d \geq 1$. Whether it can be generalized to $d \geq 2$ is an open problem. 
3. The analog of Theorem 5 for so-called space-time RWRE is proved in [22].

4. Related results have been obtained for random walks in random potentials, cf. $[9,26]$, for directed polymers in random environments, cf. [5], and for random walks on Galton-Watson trees, cf. [1,6,7].

\section{Proof of the main result}

\subsection{Outline}

For every $\theta \in \mathbb{R}^{d}$, consider the logarithmic moment generating functions

$$
\begin{aligned}
& \Lambda_{q}(\theta):=\lim _{n \rightarrow \infty} \frac{1}{n} \log E_{o}^{\omega}\left[\exp \left\{\left\langle\theta, X_{n}\right\rangle\right\}\right] \text { and } \\
& \Lambda_{a}(\theta):=\lim _{n \rightarrow \infty} \frac{1}{n} \log E_{o}\left[\exp \left\{\left\langle\theta, X_{n}\right\rangle\right\}\right] .
\end{aligned}
$$

By Varadhan's Lemma, cf. [8], $\Lambda_{q}(\theta)=\sup _{\xi \in \mathbb{R}^{d}}\left\{\langle\theta, \xi\rangle-I_{q}(\xi)\right\}=I_{q}^{*}(\theta)$, the convex conjugate of $I_{q}$ at $\theta$. Similarly, $\Lambda_{a}(\theta)=I_{a}^{*}(\theta)$.

Assume $d \geq 4$ and (T, $\hat{u}$ ) for some $\hat{u} \in \mathcal{S}^{d-1}$. For every $n \geq 0, \theta \in \mathbb{R}^{d}$ and $\omega \in \Omega$, define

$$
\begin{gathered}
H_{n}=H_{n}(\hat{u}):=\inf \left\{i \geq 0:\left\langle X_{i}, \hat{u}\right\rangle \geq n\right\}, \\
\beta=\beta(\hat{u}):=\inf \left\{i \geq 0:\left\langle X_{i}, \hat{u}\right\rangle<\left\langle X_{o}, \hat{u}\right\rangle\right\} \quad \text { and } \\
g_{n}(\theta, \omega):=E_{o}^{\omega}\left[\exp \left\{\left\langle\theta, X_{H_{n}}\right\rangle-\Lambda_{a}(\theta) H_{n}\right\}, H_{n}=\tau_{k} \text { for some } k \geq 1, \beta=\infty\right] .
\end{gathered}
$$

When $|\theta|$ is sufficiently small (and $\Lambda_{a}(\theta)>0$ in the nestling case), we show that $\left(g_{n}(\theta, \cdot)\right)_{n \geq 1}$ is bounded in $L^{2}(\mathbb{P})$ and $\mathbb{E}\left\{g_{n}(\theta, \cdot)\right\}$ converges to a nonzero limit as $n \rightarrow \infty$. These two facts imply that $\Lambda_{q}(\theta)=\Lambda_{a}(\theta)$.

Section 3 is devoted to the $L^{2}$ estimate regarding $\left(g_{n}(\theta, \cdot)\right)_{n \geq 1}$ which constitutes the core of this paper. Assuming that, the equality of the logarithmic moment generating functions is established in Sect. 2.3. Finally, convex duality is used in Sect. 2.4 to prove Theorem 5 by showing that the local equality of $\Lambda_{q}$ and $\Lambda_{a}$ implies the equality of $I_{q}$ and $I_{a}$ on certain subsets of $\mathcal{D}$.

We find it more convenient to work with regeneration times relative to a $z \in U$ rather than any $\hat{u} \in \mathcal{S}^{d-1}$. In Sect. 2.2, we give some results which imply that there is no loss of generality in doing so.

\subsection{Some preliminaries regarding regenerations}

Assume $d \geq 2,(1.1)$ and (1.4).

Lemma 6 (Sznitman [18]) Assume $(\boldsymbol{T}, \hat{u})$ for some $\hat{u} \in \mathcal{S}^{d-1}$.

(a) $P_{o}(\beta(\hat{u})=\infty)>0$, and $\tau_{1}(\hat{u})$ has finite $P_{o}$-moments of arbitrary order.

(b) The LLN holds with a limiting velocity $\xi_{o}$ such that $\left\langle\xi_{o}, \hat{u}\right\rangle>0$.

(c) $(\boldsymbol{T}, \hat{v})$ is satisfied for every $\hat{v} \in \mathcal{S}^{d-1}$ such that $\left\langle\xi_{o}, \hat{v}\right\rangle>0$. 
Lemma 7 (Sznitman [17]) If the walk is non-nestling relative to some $\hat{u} \in \mathcal{S}^{d-1}$, then

$$
E_{o}\left[\exp \left\{\kappa_{2} \tau_{1}(\hat{u})\right\}\right]<\infty
$$

for some $\kappa_{2}>0$. In particular, $(\boldsymbol{T}, \hat{u})$ is satisfied.

Lemma 8 If the walk is non-nestling and some $\hat{v} \in \mathcal{S}^{d-1}$ satisfies $\left\langle\xi_{o}, \hat{v}\right\rangle>0$, then

$$
E_{o}\left[\exp \left\{c \tau_{1}(\hat{v})\right\}\right]<\infty
$$

for some $c>0$.

Proof Since the walk is non-nestling, (1.6) holds for some $\hat{u} \in \mathcal{S}^{d-1}$ with rational coordinates. Let $a \geq 1$ be an integer such that $a \hat{u}$ has integer coordinates. Note that $\langle x, \hat{u}\rangle>0$ if and only if $\langle x, \hat{u}\rangle \geq \frac{1}{a}$ for $x \in \mathbb{Z}^{d}$. Therefore, $\left|X_{\tau_{a k+1}(\hat{u})}\right| \geq$ $\left\langle X_{\tau_{a k+1}(\hat{u})}, \hat{u}\right\rangle>k$ for every $k \geq 1$.

For every $c, c^{\prime}>0$ and $\hat{v} \in \mathcal{S}^{d-1}$ such that $\left\langle\xi_{o}, \hat{v}\right\rangle>0$,

$$
\begin{aligned}
E_{o}\left[\exp \left\{c \tau_{1}(\hat{v})\right\}\right]= & \sum_{k=1}^{\infty} E_{o}\left[\exp \left\{c \tau_{1}(\hat{v})\right\}, \sup _{1 \leq i \leq \tau_{1}(\hat{v})}\left|X_{i}\right| \in(k-1, k]\right] \\
\leq & \sum_{k=1}^{\infty} E_{o}\left[\exp \left\{c \tau_{a k+1}(\hat{u})\right\}, \sup _{1 \leq i \leq \tau_{1}(\hat{v})}\left|X_{i}\right| \in(k-1, k]\right] \\
\leq & \sum_{k=1}^{\infty} E_{o}\left[\exp \left\{c \tau_{a k+1}(\hat{u})\right\}\left(\sup _{1 \leq i \leq \tau_{1}(\hat{v})} \exp \left\{c^{\prime}\left|X_{i}\right|\right\}\right)\right] \\
& \times \exp \left\{-c^{\prime}(k-1)\right\} \\
\leq & E_{o}\left[\sup _{1 \leq i \leq \tau_{1}(\hat{v})} \exp \left\{2 c^{\prime}\left|X_{i}\right|\right\}\right]^{1 / 2} \sum_{k=1}^{\infty} E_{o}\left[\exp \left\{2 c \tau_{a k+1}(\hat{u})\right\}\right]^{1 / 2} \\
& \times \exp \left\{-c^{\prime}(k-1)\right\} .
\end{aligned}
$$

Note that $(\mathbf{T}, \hat{u})$ is satisfied by Lemma 7. Since $\left\langle\xi_{o}, \hat{v}\right\rangle>0$, it follows from Lemma 6 that $(\mathbf{T}, \hat{v})$ is satisfied as well. Therefore, (1.5) implies that the first term in (2.2) is finite when $c^{\prime}>0$ is small enough.

It is immediate from the renewal structure that

$$
\begin{aligned}
& E_{o}\left[\exp \left\{2 c \tau_{a k+1}(\hat{u})\right\}\right]^{1 / 2} \\
& =E_{o}\left[\exp \left\{2 c \tau_{1}(\hat{u})\right\}\right]^{1 / 2} E_{o}\left[\exp \left\{2 c \tau_{1}(\hat{u})\right\} \mid \beta(\hat{u})=\infty\right]^{a k / 2} .
\end{aligned}
$$

By Lemma 7, $E_{o}\left[\exp \left\{\kappa_{2} \tau_{1}(\hat{u})\right\} \mid \beta(\hat{u})=\infty\right]<\infty$ for some $\kappa_{2}>0$. When $c>0$ is small enough,

$$
E_{o}\left[\exp \left\{2 c \tau_{1}(\hat{u})\right\} \mid \beta(\hat{u})=\infty\right]^{a / 2} \leq E_{o}\left[\exp \left\{\kappa_{2} \tau_{1}(\hat{u})\right\} \mid \beta(\hat{u})=\infty\right]^{a c / \kappa_{2}}<\mathrm{e}^{c^{\prime}}
$$

and the summation in (2.2) is finite. This implies the desired result. 
Corollary 9 Assume $(\boldsymbol{T}, \hat{u})$ for some $\hat{u} \in \mathcal{S}^{d-1}$. Since $\xi_{o} \neq 0,\left\langle\xi_{o}, z\right\rangle>0$ for some $z \in U$.

(a) $P_{o}(\beta(z)=\infty)>0$, and $\tau_{1}(z)$ has finite $P_{o}$-moments of arbitrary order.

(b) If the walk is non-nestling, then there exists a $\kappa_{3}>0$ such that

$$
E_{o}\left[\exp \left\{2 \kappa_{3} \tau_{1}(z)\right\}\right]<\infty
$$

(c) If the walk is nestling, then there exists a $\kappa_{3}>0$ such that

$$
E_{o}\left[\sup _{1 \leq i \leq \tau_{1}(z)} \exp \left\{\kappa_{3}\left|X_{i}\right|\right\}\right]<\infty
$$

2.3 Equality of the logarithmic moment generating functions

Assume $d \geq 4,(1.1),(1.4)$ and $(\mathbf{T}, \hat{u})$ for some $\hat{u} \in \mathcal{S}^{d-1}$. Since $\xi_{o} \neq 0,\left\langle\xi_{o}, z\right\rangle>0$ for some $z \in U$. Assume WLOG that $\left\langle\xi_{o}, e_{1}\right\rangle>0$. Refer to (1.2) and (2.1) for the definitions of

$$
\left(\tau_{m}\right)_{m \geq 1}=\left(\tau_{m}\left(e_{1}\right)\right)_{m \geq 1}, \quad\left(H_{n}\right)_{n \geq 0}=\left(H_{n}\left(e_{1}\right)\right)_{n \geq 0} \quad \text { and } \quad \beta=\beta\left(e_{1}\right) .
$$

Fix $\kappa_{3}$ as in Corollary 9 . For every $\kappa \in\left(0, \kappa_{3}\right]$, define

$$
\mathcal{C}_{a}(\kappa):= \begin{cases}\left\{\theta \in \mathbb{R}^{d}:|\theta|<\kappa\right\} & \text { if the walk is non-nestling } \\ \left\{\theta \in \mathbb{R}^{d}:|\theta|<\kappa, \Lambda_{a}(\theta)>0\right\} & \text { if the walk is nestling. }\end{cases}
$$

By Jensen's inequality,

$$
\begin{aligned}
\left\langle\theta, \xi_{o}\right\rangle & =\lim _{n \rightarrow \infty} \frac{1}{n} E_{o}\left[\left\langle\theta, X_{n}\right\rangle\right] \leq \lim _{n \rightarrow \infty} \frac{1}{n} \log E_{o}\left[\exp \left\{\left\langle\theta, X_{n}\right\rangle\right\}\right] \\
& =\Lambda_{a}(\theta) \leq \lim _{n \rightarrow \infty} \frac{1}{n} \log E_{o}\left[\mathrm{e}^{|\theta| n}\right]=|\theta| .
\end{aligned}
$$

In the nestling case, $\left\{\theta \in \mathbb{R}^{d}:|\theta|<\kappa,\left\langle\theta, \xi_{o}\right\rangle>0\right\} \subset \mathcal{C}_{a}(\kappa)$ by (2.4). Hence, $\mathcal{C}_{a}(\kappa)$ is a non-empty open set both for nestling and non-nestling walks.

Lemma $10 E_{o}\left[\exp \left\{\left\langle\theta, X_{\tau_{1}}\right\rangle-\Lambda_{a}(\theta) \tau_{1}\right\} \mid \beta=\infty\right]=1$ for every $\theta \in \mathcal{C}_{a}\left(\kappa_{3}\right)$.

Proof This is Lemma 12 of [21].

For every $\theta \in \mathcal{C}_{a}\left(\kappa_{3}\right)$ and $y \in \mathbb{Z}^{d}$, let

$$
q^{\theta}(y):=E_{o}\left[\exp \left\{\left\langle\theta, X_{\tau_{1}}\right\rangle-\Lambda_{a}(\theta) \tau_{1}\right\}, X_{\tau_{1}}=y \mid \beta=\infty\right]
$$


Since $\sum_{y \in \mathbb{Z}^{d}} q^{\theta}(y)=1$ by Lemma $10,\left(q^{\theta}(y)\right)_{y \in \mathbb{Z}^{d}}$ defines a random walk $\left(Y_{k}\right)_{k \geq 0}$ on $\mathbb{Z}^{d}$. For every $x \in \mathbb{Z}^{d}$, this walk induces a probability measure $\hat{P}_{x}^{\theta}$ on paths starting at $x$. As usual, $\hat{E}_{x}^{\theta}$ denotes the corresponding expectation. It follows from Corollary 9 and Hölder's inequality that

$$
\hat{E}_{o}^{\theta}\left[\left|Y_{1}\right|^{m}\right]<\infty \text { for every } m \geq 1 .
$$

For every $n \geq 1, \theta \in \mathcal{C}_{a}\left(\kappa_{3}\right)$ and $\omega \in \Omega$, recall from Sect. 2.1 that $g_{n}(\theta, \omega):=E_{o}^{\omega}\left[\exp \left\{\left\langle\theta, X_{H_{n}}\right\rangle-\Lambda_{a}(\theta) H_{n}\right\}, H_{n}=\tau_{k}\right.$ for some $\left.k \geq 1, \beta=\infty\right]$.

Lemma 11 For every $\theta \in \mathcal{C}_{a}\left(\kappa_{3}\right)$,

$$
\lim _{n \rightarrow \infty} \mathbb{E}\left\{g_{n}(\theta, \cdot)\right\}=P_{o}(\beta=\infty) / \hat{E}_{o}^{\theta}\left[\left\langle Y_{1}, e_{1}\right\rangle\right]>0 .
$$

Proof For every $n \geq 1$ and $\theta \in \mathcal{C}_{a}\left(\kappa_{3}\right)$,

$$
\begin{aligned}
\mathbb{E}\left\{g_{n}(\theta, \cdot)\right\} & =E_{o}\left[\exp \left\{\left\langle\theta, X_{H_{n}}\right\rangle-\Lambda_{a}(\theta) H_{n}\right\}, H_{n}=\tau_{k} \text { for some } k \geq 1, \beta=\infty\right] \\
& =P_{o}(\beta=\infty) \sum_{k=1}^{\infty} E_{o}\left[\exp \left\{\left\langle\theta, X_{H_{n}}\right\rangle-\Lambda_{a}(\theta) H_{n}\right\}, H_{n}=\tau_{k} \mid \beta=\infty\right] \\
& =P_{o}(\beta=\infty) \sum_{k=1}^{\infty} E_{o}\left[\exp \left\{\left\langle\theta, X_{\tau_{k}}\right\rangle-\Lambda_{a}(\theta) \tau_{k}\right\},\left\langle X_{\tau_{k}}, e_{1}\right\rangle=n \mid \beta=\infty\right] \\
& =P_{o}(\beta=\infty) \sum_{k=1}^{\infty} \hat{P}_{o}^{\theta}\left(\left\langle Y_{k}, e_{1}\right\rangle=n\right) .
\end{aligned}
$$

Note that $\hat{P}_{o}^{\theta}\left(\left\langle Y_{1}, e_{1}\right\rangle=1\right)>0$ by (1.1) and part (a) of Lemma 6 . Hence, the desired result follows from the renewal theorem for aperiodic sequences, cf. Theorem 10.8 of [3].

Lemma 12 There exists a $\kappa_{e q} \in\left(0, \kappa_{3}\right)$ such that

$$
\sup _{n \geq 1} \mathbb{E}\left\{g_{n}(\theta, \cdot)^{2}\right\}<\infty
$$

for every $\theta \in \mathcal{C}_{a}\left(\kappa_{e q}\right)$.

Remark 13 Lemma 12 is proved in Sect. 3.

Lemma 14 For every $\theta \in \mathcal{C}_{a}\left(\kappa_{e q}\right)$,

$$
\mathbb{P}\left\{\omega: \lim _{n \rightarrow \infty} g_{n}(\theta, \omega)=0\right\}<1 .
$$


Proof Take any $\theta \in \mathcal{C}_{a}\left(\kappa_{e q}\right)$. Note that $\left(g_{n}(\theta, \cdot)\right)_{n \geq 1}$ is uniformly integrable by Lemma 12. If $g_{n}(\theta, \cdot)$ were to converge $\mathbb{P}$-a.s. to 0 as $n \rightarrow \infty$, then $\lim _{n \rightarrow \infty} \mathbb{E}\left\{g_{n}(\theta, \cdot)\right\}=0$ would hold. However, this would contradict Lemma 11.

Lemma 15 For every $\theta \in \mathbb{R}^{d}, \epsilon>0$ and $\mathbb{P}$-a.e. $\omega$,

$$
\lim _{n \rightarrow \infty} E_{o}^{\omega}\left[\exp \left\{\left\langle\theta, X_{H_{n}}\right\rangle-\left(\Lambda_{q}(\theta)+\epsilon\right) H_{n}\right\}\right]=0
$$

Proof For every $n \geq 1, \theta \in \mathbb{R}^{d}, \epsilon>0$ and $\mathbb{P}$-a.e. $\omega$,

$$
\begin{aligned}
& E_{o}^{\omega}\left[\exp \left\{\left\langle\theta, X_{H_{n}}\right\rangle-\left(\Lambda_{q}(\theta)+\epsilon\right) H_{n}\right\}\right] \\
& =\sum_{i=n}^{\infty} E_{o}^{\omega}\left[\exp \left\{\left\langle\theta, X_{H_{n}}\right\rangle-\left(\Lambda_{q}(\theta)+\epsilon\right) H_{n}\right\}, H_{n}=i\right] \\
& \leq \sum_{i=n}^{\infty} E_{o}^{\omega}\left[\exp \left\{\left\langle\theta, X_{i}\right\rangle-\left(\Lambda_{q}(\theta)+\epsilon\right) i\right\}\right]=\sum_{i=n}^{\infty} \mathrm{e}^{o(i)-\epsilon i} \leq \sum_{i=n}^{\infty} \mathrm{e}^{-\epsilon i / 2}
\end{aligned}
$$

when $n$ is sufficiently large. Therefore,

$\limsup _{n \rightarrow \infty} E_{o}^{\omega}\left[\exp \left\{\left\langle\theta, X_{H_{n}}\right\rangle-\left(\Lambda_{q}(\theta)+\epsilon\right) H_{n}\right\}\right] \leq \limsup _{n \rightarrow \infty} \mathrm{e}^{-\epsilon n / 2}\left(1-\mathrm{e}^{-\epsilon / 2}\right)^{-1}=0$.

Lemma $16 \Lambda_{q}(\theta)=\Lambda_{a}(\theta)$ for every $\theta \in \mathcal{C}_{a}\left(\kappa_{e q}\right)$.

Proof For every $\theta \in \mathbb{R}^{d}$, it follows from Jensen's inequality and the bounded convergence theorem that

$$
\begin{aligned}
\Lambda_{q}(\theta) & =\mathbb{E}\left\{\lim _{n \rightarrow \infty} \frac{1}{n} \log E_{o}^{\omega}\left[\exp \left\{\left\langle\theta, X_{n}\right\rangle\right\}\right]\right\}=\lim _{n \rightarrow \infty} \frac{1}{n} \mathbb{E}\left\{\log E_{o}^{\omega}\left[\exp \left\{\left\langle\theta, X_{n}\right\rangle\right\}\right]\right\} \\
& \leq \lim _{n \rightarrow \infty} \frac{1}{n} \log E_{o}\left[\exp \left\{\left\langle\theta, X_{n}\right\rangle\right\}\right]=\Lambda_{a}(\theta) .
\end{aligned}
$$

Let us now establish the reverse inequality. For every $\theta \in \mathcal{C}_{a}\left(\kappa_{e q}\right)$ and $\epsilon>0$,

$$
\begin{aligned}
& \mathbb{P}\left\{\omega: \lim _{n \rightarrow \infty} E_{o}^{\omega}\left[\exp \left\{\left\langle\theta, X_{H_{n}}\right\rangle-\Lambda_{a}(\theta) H_{n}\right\}\right]=0\right\}<1 \text { and } \\
& \mathbb{P}\left\{\omega: \lim _{n \rightarrow \infty} E_{o}^{\omega}\left[\exp \left\{\left\langle\theta, X_{H_{n}}\right\rangle-\left(\Lambda_{q}(\theta)+\epsilon\right) H_{n}\right\}\right]=0\right\}=1
\end{aligned}
$$

by Lemmas 14 and 15 , respectively. Therefore, $\Lambda_{q}(\theta)+\epsilon>\Lambda_{a}(\theta)$. Since $\epsilon>0$ is arbitrary, we conclude that $\Lambda_{q}(\theta) \geq \Lambda_{a}(\theta)$ for every $\theta \in \mathcal{C}_{a}\left(\kappa_{e q}\right)$. 
2.4 Equality of the rate functions

Since $\Lambda_{q}=\Lambda_{a}$ on $\mathcal{C}_{a}\left(\kappa_{e q}\right)$, it will follow from convex duality that $I_{q}(\xi)=I_{a}(\xi)$ for every $\xi \in \mathcal{D}$ that defines a supporting hyperplane of $\Lambda_{a}$ at some $\theta \in \mathcal{C}_{a}\left(\kappa_{e q}\right)$. In order to show that the set of such $\xi$ satisfies the properties stated in Theorem 5, we need two preliminary lemmas.

Lemma 17 Assume that the walk is nestling. Define

$$
\mathcal{C}_{a}^{b}\left(\kappa_{e q}\right):=\left\{\theta \in \partial \mathcal{C}_{a}\left(\kappa_{e q}\right):|\theta|<\kappa_{e q}\right\}
$$

(a) If $|\theta|<\kappa_{e q}$, then $\theta \notin \mathcal{C}_{a}\left(\kappa_{e q}\right)$ if and only if $E_{o}\left[\exp \left\{\left\langle\theta, X_{\tau_{1}}\right\rangle\right\} \mid \beta=\infty\right] \leq 1$.

(b) If $|\theta|<\kappa_{e q}$, then $\theta \in \mathcal{C}_{a}^{b}\left(\kappa_{e q}\right)$ if and only if $E_{o}\left[\exp \left\{\left\langle\theta, X_{\tau_{1}}\right\rangle\right\} \mid \beta=\infty\right]=1$.

Proof This is Lemma 13 of [21].

Lemma $18 \Lambda_{a}$ is analytic on $\mathcal{C}_{a}\left(\kappa_{e q}\right)$. Its gradient $\nabla \Lambda_{a}$ extends smoothly to $\overline{\mathcal{C}_{a}\left(\kappa_{e q}\right)}$, the closure of $\mathcal{C}_{a}\left(\kappa_{e q}\right)$. Moreover, the extension of the Hessian $\mathcal{H}_{a}$ of $\Lambda_{a}$ is positive definite on $\overline{\mathcal{C}_{a}\left(\kappa_{e q}\right)}$.

Proof This follows immediately from the proof of Lemma 6 of [21].

Proof of Theorem 5 (a) The non-nestling case: Recall that $\Lambda_{a}$ is analytic on $\mathcal{C}_{a}\left(\kappa_{e q}\right)$. Define $\mathcal{A}_{e q}:=\left\{\nabla \Lambda_{a}(\theta): \theta \in \mathcal{C}_{a}\left(\kappa_{e q}\right)\right\} . \nabla \Lambda_{a}: \mathcal{C}_{a}\left(\kappa_{e q}\right) \rightarrow \mathcal{A}_{e q}$ is invertible since the Hessian $\mathcal{H}_{a}$ of $\Lambda_{a}$ is positive definite on $\mathcal{C}_{a}\left(\kappa_{e q}\right)$. The inverse, denoted by $\Gamma_{a}: \mathcal{A}_{e q} \rightarrow \mathcal{C}_{a}\left(\kappa_{e q}\right)$, is analytic by the inverse function theorem (cf. Theorem 6.1.2 of [11]), and $\mathcal{A}_{e q}$ is open.

For every $\xi \in \mathcal{A}_{\text {eq }}$,

$$
I_{a}(\xi)=\sup _{\theta \in \mathbb{R}^{d}}\left\{\langle\theta, \xi\rangle-\Lambda_{a}(\theta)\right\}=\left\langle\Gamma_{a}(\xi), \xi\right\rangle-\Lambda_{a}\left(\Gamma_{a}(\xi)\right)
$$

Thus, $I_{a}$ is analytic on $\mathcal{A}_{e q}$. Differentiating (2.9) twice with respect to $\xi$ shows that the Hessian of $I_{a}$ at $\xi$ is equal to $\mathcal{H}_{a}\left(\Gamma_{a}(\xi)\right)^{-1}$, a positive definite matrix. Therefore, $I_{a}$ is strictly convex on $\mathcal{A}_{e q}$.

It is shown in [19] that $\xi_{o}=\left(E_{o}\left[X_{\tau_{1}} \mid \beta=\infty\right]\right) /\left(E_{o}\left[\tau_{1} \mid \beta=\infty\right]\right)$. Since $0 \in$ $\mathcal{C}_{a}\left(\kappa_{e q}\right)$, it follows that $\xi_{o}=\nabla \Lambda_{a}(0) \in \mathcal{A}_{e q}$.

$\Lambda_{q}=\Lambda_{a}$ on $\mathcal{C}_{a}\left(\kappa_{e q}\right)$ by Lemma 16 . For every $\xi \in \mathcal{A}_{e q}$,

$$
I_{q}(\xi)=\sup _{\theta \in \mathbb{R}^{d}}\left\{\langle\theta, \xi\rangle-\Lambda_{q}(\theta)\right\}=\left\langle\Gamma_{a}(\xi), \xi\right\rangle-\Lambda_{a}\left(\Gamma_{a}(\xi)\right)=I_{a}(\xi) .
$$

(b) The nestling case: Recall that $\nabla \Lambda_{a}$ extends smoothly to $\overline{\mathcal{C}_{a}\left(\kappa_{e q}\right)}$. Refer to the extension by $\overline{\nabla \Lambda_{a}}$. Define $\mathcal{A}_{e q}^{+}:=\left\{\nabla \Lambda_{a}(\theta): \theta \in \mathcal{C}_{a}\left(\kappa_{e q}\right)\right\}$ and $\mathcal{A}_{e q}^{b}:=$ $\left\{\overline{\nabla \Lambda_{a}}(\theta): \theta \in \mathcal{C}_{a}^{b}\left(\kappa_{e q}\right)\right\}$ with $\mathcal{C}_{a}^{b}\left(\kappa_{e q}\right)$ as in (2.8). Note that $0 \in \mathcal{C}_{a}^{b}\left(\kappa_{e q}\right) \subset$ $\partial \mathcal{C}_{a}\left(\kappa_{e q}\right)$ by Lemma 17 , and $\xi_{o}=\overline{\nabla \Lambda_{a}}(0) \in \mathcal{A}_{e q}^{b} \subset \partial \mathcal{A}_{e q}^{+}$. 
Similar to the non-nestling case, $I_{a}$ is strictly convex and analytic on $\mathcal{A}_{e q}^{+}$which is an open set, and $I_{q}(\xi)=I_{a}(\xi)$ for every $\xi \in \mathcal{A}_{e q}^{+}$. Moreover, $\mathcal{A}_{e q}^{b}$ is a $(d-1)$ dimensional smooth surface patch and item (iii) is satisfied. (The latter facts follow from part (d) of Theorem 3 since $\mathcal{A}_{e q}^{b} \subset \mathcal{A}_{a}^{b}$, cf. [21].)

It remains to show that $I_{q}(t \xi)=t I_{q}(\xi)=t I_{a}(\xi)=I_{a}(t \xi)$ for every $\xi \in \mathcal{A}_{e q}^{b}$ and $t \in[0,1]$. The rest of this proof focuses on this statement.

For every $\xi \in \mathcal{A}_{e q}^{b}$, there exists a $\theta \in \mathcal{C}_{a}^{b}\left(\kappa_{e q}\right)$ such that $\xi=\overline{\nabla \Lambda_{a}}(\theta)$ and

$$
\left\langle\xi, e_{1}\right\rangle=\left\langle\overline{\nabla \Lambda_{a}}(\theta), e_{1}\right\rangle=\frac{E_{o}\left[\left\langle X_{\tau_{1}}, e_{1}\right\rangle \exp \left\{\left\langle\theta, X_{\tau_{1}}\right\rangle\right\} \mid \beta=\infty\right]}{E_{o}\left[\tau_{1} \exp \left\{\left\langle\theta, X_{\tau_{1}}\right\rangle\right\} \mid \beta=\infty\right]}>0
$$

Suppose $\xi=\overline{\nabla \Lambda_{a}}\left(\theta^{\prime}\right)$ for some $\theta^{\prime} \in \mathcal{C}_{a}^{b}\left(\kappa_{e q}\right)$ such that $\theta \neq \theta^{\prime}$. Then, for every $t \in(0,1), \xi$ defines a supporting hyperplane of $\Lambda_{a}$ at $\theta_{t}:=t \theta+(1-t) \theta^{\prime}$. Recall Lemma 17. $E_{o}\left[\exp \left\{\left\langle\theta_{t}, X_{\tau_{1}}\right\rangle\right\} \mid \beta=\infty\right]<1$ by Jensen's inequality, and $\theta_{t}$ is an interior point of $\mathcal{C}_{a}\left(\kappa_{e q}\right)^{c}$. Therefore, $\nabla \Lambda_{a}\left(\theta_{t}\right)=0$ since $\Lambda_{a}$ is identically equal to zero on $\left\{\theta:|\theta|<\kappa_{e q}\right\} \backslash \mathcal{C}_{a}\left(\kappa_{e q}\right)$. However, this contradicts (2.10). We conclude that there exists a unique $\theta \in \mathcal{C}_{a}^{b}\left(\kappa_{e q}\right)$ such that $\xi=\overline{\nabla \Lambda_{a}}(\theta)$. Denote the inverse of $\overline{\nabla \Lambda_{a}}$ by $\overline{\Gamma_{a}}$.

For every $\xi \in \mathcal{A}_{e q}^{b}$ and $t \in[0,1], \exists \theta_{n} \in \mathcal{C}_{a}\left(\kappa_{e q}\right)$ such that $\theta_{n} \rightarrow \overline{\Gamma_{a}}(\xi)$ and $\xi_{n}:=\nabla \Lambda_{a}\left(\theta_{n}\right) \rightarrow \xi$ as $n \rightarrow \infty$. Note that $\Lambda_{a}\left(\overline{\Gamma_{a}}(\xi)\right)=0$ since $\overline{\Gamma_{a}}(\xi) \in \mathcal{C}_{a}^{b}\left(\kappa_{e q}\right)$. By the continuity of $I_{a}$ and $\Lambda_{a}$,

$$
\begin{gathered}
I_{a}(\xi)=\lim _{n \rightarrow \infty} I_{a}\left(\xi_{n}\right)=\lim _{n \rightarrow \infty}\left\langle\theta_{n}, \xi_{n}\right\rangle-\Lambda_{a}\left(\theta_{n}\right)=\left\langle\overline{\Gamma_{a}}(\xi), \xi\right\rangle-\Lambda_{a}\left(\overline{\Gamma_{a}}(\xi)\right)=\left\langle\overline{\Gamma_{a}}(\xi), \xi\right\rangle \text { and } \\
I_{a}(t \xi)=\sup _{\theta \in \mathbb{R}^{d}}\left\{\langle\theta, t \xi\rangle-\Lambda_{a}(\theta)\right\} \geq\left\langle\overline{\Gamma_{a}}(\xi), t \xi\right\rangle-\Lambda_{a}\left(\overline{\Gamma_{a}}(\xi)\right)=t\left\langle\overline{\Gamma_{a}}(\xi), \xi\right\rangle=t I_{a}(\xi) .
\end{gathered}
$$

Conversely, $I_{a}(t \xi) \leq t I_{a}(\xi)+(1-t) I_{a}(0)=t I_{a}(\xi)$ by Jensen's inequality (and the fact that $I_{a}(0)=0$, cf. Theorem 3$)$. Hence, $I_{a}(t \xi)=t I_{a}(\xi)$.

The continuity of the rate functions implies that $I_{q}=I_{a}$ on $\mathcal{A}_{e q}^{b}$. Recall that $I_{q}(0)=0$, cf. Theorem 3 . Since the averaged rate function is always less than or equal to the quenched rate function, we conclude that

$$
I_{q}(t \xi) \leq t I_{q}(\xi)+(1-t) I_{q}(0)=t I_{q}(\xi)=t I_{a}(\xi)=I_{a}(t \xi) \leq I_{q}(t \xi)
$$

Remark 19 The argument above, due to its structure, not only proves Theorem 5, but also reproduces some of the proofs of the statements in Theorem 3 that are given in [21]. Moreover, it provides a new and concise proof of item (v) of part (d) of Theorem 3 which is originally obtained in [12].

\section{The $L^{2}$ estimate}

In our proof of Theorem 5 given in Sect. 2, we assumed Lemma 12. In this section, we will verify this assumption. The following fact will play a central role in our argument: 
if the dimension is at least four, then, with positive averaged probability, the paths of two independent ballistic walks in the same environment do not intersect.

\subsection{Some preliminaries regarding two walks}

Assume $d \geq 4,(1.1),(1.4)$ and $(\mathbf{T}, \hat{u})$ for some $\hat{u} \in \mathcal{S}^{d-1}$. Also, like in Sects. 2.3 and 2.4, assume WLOG that $\left\langle\xi_{o}, e_{1}\right\rangle>0$.

For every $x$ and $\tilde{x} \in \mathbb{Z}^{d}$, consider two independent walks $X=X(x):=\left(X_{i}\right)_{i \geq 0}$ and $\tilde{X}=\tilde{X}(\tilde{x}):=\left(\tilde{X}_{j}\right)_{j \geq 0}$ starting at $x$ and $\tilde{x}$ in the same environment. Denote their joint quenched law and joint averaged law by $P_{x, \tilde{x}}^{\omega}:=P_{x}^{\omega} \times P_{\tilde{x}}^{\omega}$ and $P_{x, \tilde{x}}(\cdot):=\mathbb{E}\left\{P_{x, \tilde{x}}^{\omega}(\cdot)\right\}$. As usual, $E_{x, \tilde{x}}^{\omega}$ and $E_{x, \tilde{x}}$ refer to expectations under $P_{x, \tilde{x}}^{\omega}$ and $P_{x, \tilde{x}}$, respectively.

Clearly, $P_{x, \tilde{x}} \neq P_{x} \times P_{\tilde{x}}$. On the other hand, the two walks don't know that they are in the same environment unless their paths intersect. In particular, for any event $A$ involving $X$ and $\tilde{X}$,

$$
P_{x, \tilde{x}}\left(A \cap\left\{v_{1}=\infty\right\}\right)=P_{x} \times P_{\tilde{x}}\left(A \cap\left\{v_{1}=\infty\right\}\right)
$$

where

$$
\nu_{1}:=\inf \left\{m \in \mathbb{Z}: X_{i}=\tilde{X}_{j} \text { for some } i \geq 0, j \geq 0, \text { and }\left\langle X_{i}, e_{1}\right\rangle=m\right\}
$$

Similar to the random times $\left(\tau_{m}\right)_{m \geq 1}=\left(\tau_{m}\left(e_{1}\right)\right)_{m \geq 1},\left(H_{n}\right)_{n \geq 0}=\left(H_{n}\left(e_{1}\right)\right)_{n \geq 0}$ and $\beta=\beta\left(e_{1}\right)$ defined in (1.2) and (2.1) for $X$, consider $\left(\tilde{\tau}_{m}\right)_{m \geq 1}=\left(\tilde{\tau}_{m}\left(e_{1}\right)\right)_{m \geq 1}$, $\left(\tilde{H}_{n}\right)_{n \geq 0}=\left(\tilde{H}_{n}\left(e_{1}\right)\right)_{n \geq 0}$ and $\tilde{\beta}=\tilde{\beta}\left(e_{1}\right)$ for $\tilde{X}$. In our proof of Lemma 12, we will make use of the joint regeneration levels of $X$ and $\tilde{X}$, which are elements of

$$
\mathcal{L}:=\left\{n \geq 0:\left\langle X_{i}, e_{1}\right\rangle \geq n \text { and }\left\langle\tilde{X}_{j}, e_{1}\right\rangle \geq n \text { for every } i \geq H_{n} \text { and } j \geq \tilde{H}_{n}\right\}
$$

This random set has been previously introduced and studied by Rassoul-Agha and Seppäläinen [14]. Note that if the starting points $x$ and $\tilde{x}$ are both in $\mathbb{V}_{d}:=$ $\left\{z \in \mathbb{Z}^{d}:\left\langle z, e_{1}\right\rangle=0\right\}$, then

$$
0 \in \mathcal{L} \Longleftrightarrow \beta=\tilde{\beta}=\infty \Longleftrightarrow l_{1}:=\inf \mathcal{L}=0
$$

Let $\mathbb{V}_{d}^{\prime}:=\mathbb{V}_{d} \backslash\{0\}$. As mentioned in the opening paragraph of this section, the following lemma is central to our proof of Lemma 12.

Lemma 20 (Berger and Zeitouni [2], Proposition 3.4)

$$
\inf _{z \in \mathbb{V}_{d}^{\prime}} P_{o, z}\left(l_{1}=0\right) \geq \inf _{z \in \mathbb{V}_{d}^{\prime}} P_{o, z}\left(v_{1}=\infty, l_{1}=0\right)>0
$$

The proof of Lemma 20 is based on certain Green's function estimates which fail to hold unless $d \geq 4$. 


\subsection{A renewal argument}

For every $n \geq 1, \theta \in \mathcal{C}_{a}\left(\kappa_{3}\right), z \in \mathbb{V}_{d}$ and $\omega \in \Omega$,

$$
\begin{aligned}
g_{n}(\theta, \omega) & =E_{o}^{\omega}\left[\exp \left\{\left\langle\theta, X_{H_{n}}\right\rangle-\Lambda_{a}(\theta) H_{n}\right\}, H_{n}=\tau_{k} \text { for some } k \geq 1, \beta=\infty\right] \text { and } \\
g_{n}\left(\theta, T_{z} \omega\right) & =E_{o}^{T_{z} \omega}\left[\exp \left\{\left\langle\theta, X_{H_{n}}\right\rangle-\Lambda_{a}(\theta) H_{n}\right\}, H_{n}=\tau_{k} \text { for some } k \geq 1, \beta=\infty\right] \\
& =\mathrm{e}^{-\langle\theta, z\rangle} E_{z}^{\omega}\left[\exp \left\{\left\langle\theta, X_{H_{n}}\right\rangle-\Lambda_{a}(\theta) H_{n}\right\}, H_{n}=\tau_{k} \text { for some } k \geq 1, \beta=\infty\right] .
\end{aligned}
$$

Thus,

$$
G_{n, z}(\theta):=\mathbb{E}\left\{g_{n}(\theta, \cdot) g_{n}\left(\theta, T_{z} \cdot\right)\right\}=\mathrm{e}^{-\langle\theta, z\rangle} E_{o, z}\left[f(\theta, n, X, \tilde{X}), n \in \mathcal{L}, l_{1}=0\right] \text { where }
$$
$f(\theta, n, X, \tilde{X}):=\exp \left\{\left\langle\theta, X_{H_{n}}\right\rangle-\Lambda_{a}(\theta) H_{n}\right\} \exp \left\{\left\langle\theta, \tilde{X}_{\tilde{H}_{n}}\right\rangle-\Lambda_{a}(\theta) \tilde{H}_{n}\right\}$.

Our aim is to show that $\left(G_{n, o}(\theta)\right)_{n \geq 1}$ is bounded. We start the argument by considering a related family of functions $\left(F_{n, z}(\theta)\right)_{n \geq 1, z \in \mathbb{V}_{d}^{\prime}}$ where

$$
F_{n, z}(\theta):=\mathrm{e}^{-\langle\theta, z\rangle} E_{o, z}\left[f(\theta, n, X, \tilde{X}), n \in \mathcal{L}, X_{H_{n}} \neq \tilde{X}_{\tilde{H}_{n}} \mid l_{1}=0\right] .
$$

Recall (3.2). It follows from the definitions and the regeneration structure that

$$
\begin{aligned}
F_{n, z}(\theta)= & \sum_{k=1}^{n} \sum_{z^{\prime} \in \mathbb{V}_{d}^{\prime}} \mathrm{e}^{-\langle\theta, z\rangle} E_{o, z}\left[f(\theta, n, X, \tilde{X}), k=\inf \left\{l \in \mathcal{L}: l>v_{1}, X_{H_{l}} \neq \tilde{X}_{\tilde{H}_{l}}\right\}\right. \\
\left.\tilde{X}_{\tilde{H}_{k}}-X_{H_{k}}=z^{\prime}, \quad n \in \mathcal{L}, X_{H_{n}} \neq \tilde{X}_{\tilde{H}_{n}} \mid l_{1}=0\right] & \\
& +\mathrm{e}^{-\langle\theta, z\rangle} E_{o, z}\left[f(\theta, n, X, \tilde{X}), n \leq v_{1}, n \in \mathcal{L}, X_{H_{n}} \neq \tilde{X}_{\tilde{H}_{n}} \mid l_{1}=0\right] \\
= & \sum_{k=1}^{n} \sum_{z^{\prime} \in \mathbb{V}_{d}^{\prime}} \mathrm{e}^{-\langle\theta, z\rangle} E_{o, z}\left[f(\theta, k, X, \tilde{X}), k=\inf \left\{l \in \mathcal{L}: l>v_{1}, X_{H_{l}} \neq \tilde{X}_{\tilde{H}_{l}}\right\}\right. \\
& \times \mathrm{e}^{-\left\langle\theta, z^{\prime}\right\rangle} E_{o, z^{\prime}}\left[f(\theta, n-k, X, \tilde{X}), n-k \in \mathcal{L}, X_{H_{n-k}} \neq \tilde{X}_{\tilde{H}_{n-k}} \mid l_{1}=0\right] \\
& +\mathrm{e}^{-\langle\theta, z\rangle} E_{o, z}\left[f(\theta, n, X, \tilde{X}), n \leq z_{1}, n \in \mathcal{L}, l_{H_{n}} \neq \tilde{X}_{\tilde{H}_{n}} \mid l_{1}=0\right] .
\end{aligned}
$$

Therefore,

$$
\begin{aligned}
F_{n, z}(\theta) \leq & \sum_{k=1}^{n} \mathrm{e}^{-\langle\theta, z\rangle} E_{o, z}\left[f(\theta, k, X, \tilde{X}), k=\inf \left\{l \in \mathcal{L}: l>v_{1}, X_{H_{l}} \neq \tilde{X}_{\tilde{H}_{l}}\right\} \mid l_{1}=0\right] \\
& \times \sup _{z^{\prime} \in \mathbb{V}_{d}^{\prime}} F_{n-k, z^{\prime}}(\theta) \\
& +\mathrm{e}^{-\langle\theta, z\rangle} E_{o, z}\left[f(\theta, n, X, \tilde{X}), n \leq v_{1}, n \in \mathcal{L}, X_{H_{n}} \neq \tilde{X}_{\tilde{H}_{n}} \mid l_{1}=0\right] .
\end{aligned}
$$


In other words,

$$
F_{n, z}(\theta) \leq \sum_{k=1}^{n} B_{k, z}(\theta) \sup _{z^{\prime} \in \mathbb{V}_{d}^{\prime}} F_{n-k, z^{\prime}}(\theta)+C_{n, z}(\theta)
$$

where

$$
\begin{aligned}
B_{k, z}(\theta) & :=\mathrm{e}^{-\langle\theta, z\rangle} E_{o, z}\left[f(\theta, k, X, \tilde{X}), k=\inf \left\{l \in \mathcal{L}: l>v_{1}, X_{H_{l}} \neq \tilde{X}_{\tilde{H}_{l}}\right\} \mid l_{1}=0\right] \text { and } \\
C_{n, z}(\theta) & :=\mathrm{e}^{-\langle\theta, z\rangle} E_{o, z}\left[f(\theta, n, X, \tilde{X}), n \leq v_{1}, n \in \mathcal{L}, X_{H_{n}} \neq \tilde{X}_{\tilde{H}_{n}} \mid l_{1}=0\right] .
\end{aligned}
$$

Lemma 21 There exists a $\kappa_{e q} \in\left(0, \kappa_{3}\right)$ such that

(a) $C(\theta):=\sup _{n \geq 1} \sup _{z \in \mathbb{V}_{d}^{\prime}} C_{n, z}(\theta)<\infty \quad$ and $\quad$ (b) $B(\theta):=\sup _{z \in \mathbb{V}_{d}^{\prime}} \sum_{k=1}^{\infty} B_{k, z}(\theta)<1$ for every $\theta \in \mathcal{C}_{a}\left(\kappa_{e q}\right)$.

Remark 22 Lemma 21 is proved in Sect. 3.3.

Lemma 23 For every $\theta \in \mathcal{C}_{a}\left(\kappa_{e q}\right)$,

$$
\sup _{n \geq 1} \sup _{z \in \mathbb{V}_{d}^{\prime}} F_{n, z}(\theta)<\infty .
$$

Proof For every $n \geq 1, N \geq n$ and $z \in \mathbb{V}_{d}^{\prime}$,

$$
\begin{aligned}
F_{n, z}(\theta) & \leq \sum_{k=1}^{n} B_{k, z}(\theta) \sup _{z^{\prime} \in \mathbb{V}_{d}^{\prime}} F_{n-k, z^{\prime}}(\theta)+C_{n, z}(\theta) \\
& \leq\left(\sum_{k=1}^{n} B_{k, z}(\theta)\right) \sup _{m \leq N} \sup _{z^{\prime} \in \mathbb{V}_{d}^{\prime}} F_{m, z^{\prime}}(\theta)+C_{n, z}(\theta) \\
& \leq B(\theta) \sup _{m \leq N} \sup _{z^{\prime} \in \mathbb{V}_{d}^{\prime}} F_{m, z^{\prime}}(\theta)+C(\theta)
\end{aligned}
$$

Therefore,

$$
\sup _{n \leq N} \sup _{z \in \mathbb{V}_{d}^{\prime}} F_{n, z}(\theta) \leq B(\theta) \sup _{n \leq N} \sup _{z \in \mathbb{V}_{d}^{\prime}} F_{n, z}(\theta)+C(\theta) .
$$

Finally, by Lemma 21,

$$
\sup _{n \geq 1} \sup _{z \in \mathbb{V}_{d}^{\prime}} F_{n, z}(\theta) \leq C(\theta)(1-B(\theta))^{-1}<\infty .
$$


Proof of Lemma 12 For every $n \geq 1, \theta \in \mathcal{C}_{a}\left(\kappa_{e q}\right)$ and $z \in \mathbb{V}_{d}^{\prime}$,

$$
\begin{aligned}
& F_{n+1, z}(\theta)=\mathrm{e}^{-\langle\theta, z\rangle} E_{o, z}\left[f(\theta, n+1, X, \tilde{X}), n+1 \in \mathcal{L}, X_{H_{n+1}} \neq \tilde{X}_{\tilde{H}_{n+1}} \mid l_{1}=0\right] \\
& \geq \mathrm{e}^{-\langle\theta, z\rangle} E_{o, z}[f(\theta, n+1, X, \tilde{X}), n \in \mathcal{L}, \\
& \left.X_{H_{n}}=\tilde{X}_{\tilde{H}_{n}}, n+1 \in \mathcal{L}, X_{H_{n+1}} \neq \tilde{X}_{\tilde{H}_{n+1}} \mid l_{1}=0\right] \\
& =\mathrm{e}^{-\langle\theta, z\rangle} E_{o, z}\left[f(\theta, n, X, \tilde{X}), n \in \mathcal{L}, X_{H_{n}}=\tilde{X}_{\tilde{H}_{n}} \mid l_{1}=0\right] \\
& \times E_{o, o}\left[f(\theta, 1, X, \tilde{X}), 1 \in \mathcal{L}, X_{H_{1}} \neq \tilde{X}_{\tilde{H}_{1}} \mid l_{1}=0\right] .
\end{aligned}
$$

Therefore,

$$
\begin{aligned}
& \frac{G_{n, z}(\theta)}{P_{o, z}\left(l_{1}=0\right)}-F_{n, z}(\theta) \\
& =\mathrm{e}^{-\langle\theta, z\rangle} E_{o, z}\left[f(\theta, n, X, \tilde{X}), n \in \mathcal{L}, X_{H_{n}}=\tilde{X}_{\tilde{H}_{n}} \mid l_{1}=0\right] \\
& \leq E_{o, o}\left[f(\theta, 1, X, \tilde{X}), 1 \in \mathcal{L}, X_{H_{1}} \neq \tilde{X}_{\tilde{H}_{1}} \mid l_{1}=0\right]^{-1} F_{n+1, z}(\theta) .
\end{aligned}
$$

By the uniform ellipticity assumption (1.1), Lemma 20, and part (a) of Lemma 6, the first term in (3.3) is bounded from above. This, in combination with Lemma 23, implies that

$$
\sup _{n \geq 1} \sup _{z \in \mathbb{V}_{d}^{\prime}} G_{n, z}(\theta)<\infty \text {. }
$$

For every $\hat{z} \in U \cap \mathbb{V}_{d}$,

$$
\begin{aligned}
g_{n}\left(\theta, T_{\hat{z}} \omega\right) & =\mathrm{e}^{-\langle\theta, \hat{z}\rangle} E_{\hat{z}}^{\omega}\left[\exp \left\{\left\langle\theta, X_{H_{n}}\right\rangle-\Lambda_{a}(\theta) H_{n}\right\}, H_{n}=\tau_{k} \text { for some } k \geq 1, \beta=\infty\right] \\
& \geq \mathrm{e}^{-\langle\theta, \hat{z}\rangle} E_{\hat{z}}^{\omega}\left[\exp \left\{\left\langle\theta, X_{H_{n}}\right\rangle-\Lambda_{a}(\theta) H_{n}\right\}, H_{n}=\tau_{k} \text { for some } k \geq 1, X_{1}=0, \beta=\infty\right] \\
& \geq \delta \mathrm{e}^{-\langle\theta, \hat{z}\rangle-\Lambda_{a}(\theta)} E_{o}^{\omega}\left[\exp \left\{\left\langle\theta, X_{H_{n}}\right\rangle-\Lambda_{a}(\theta) H_{n}\right\}, H_{n}=\tau_{k} \text { for some } k \geq 1, \beta=\infty\right] \\
& =\delta \mathrm{e}^{-\langle\theta, \hat{z}\rangle-\Lambda_{a}(\theta)} g_{n}(\theta, \omega) \geq \delta \mathrm{e}^{-2 \kappa_{e q}} g_{n}(\theta, \omega) .
\end{aligned}
$$

Hence,

$$
\begin{aligned}
\sup _{n \geq 1} \mathbb{E}\left\{g_{n}(\theta, \cdot)^{2}\right\} & \leq \delta^{-1} \mathrm{e}^{2 \kappa_{e q}} \sup _{n \geq 1} \sup _{z \in \mathbb{V}_{d}^{\prime}} \mathbb{E}\left\{g_{n}(\theta, \cdot) g_{n}\left(\theta, T_{z} \cdot\right)\right\} \\
& =\delta^{-1} \mathrm{e}^{2 \kappa_{e q}} \sup _{n \geq 1} \sup _{z \in \mathbb{V}_{d}^{\prime}} G_{n, z}(\theta)<\infty
\end{aligned}
$$




\subsection{Proof of Lemma 21}

Let us start by proving the easy part.

Proof of part (a) of Lemma 21 For every $n \geq 1, \theta \in \mathcal{C}_{a}\left(\kappa_{3}\right)$ and $z \in \mathbb{V}_{d}^{\prime}$,

$$
\begin{gathered}
C_{n, z}(\theta) \leq \frac{\mathrm{e}^{-\langle\theta, z\rangle}}{P_{o, z}\left(l_{1}=0\right)} E_{o, z}\left[f(\theta, n, X, \tilde{X}), n \leq v_{1}, n \in \mathcal{L}, \beta=\infty, \tilde{\beta}=\infty\right] \\
\leq \frac{\mathrm{e}^{-\langle\theta, z\rangle}}{P_{o, z}\left(l_{1}=0\right)} E_{o, z}\left[f(\theta, n, X, \tilde{X}),\left\{X_{i}: 0 \leq i<H_{n}\right\} \cap\left\{\tilde{X}_{j}: 0 \leq j<\tilde{H}_{n}\right\}=\emptyset\right. \\
\left.\quad \beta \geq H_{n}, \tilde{\beta} \geq \tilde{H}_{n}\right] \\
=\frac{\mathrm{e}^{-\langle\theta, z\rangle}}{P_{o, z}\left(l_{1}=0\right)} E_{o} \times E_{z}\left[f(\theta, n, X, \tilde{X}),\left\{X_{i}: 0 \leq i<H_{n}\right\} \cap\left\{\tilde{X}_{j}: 0 \leq j<\tilde{H}_{n}\right\}=\emptyset\right. \\
\left.\quad \quad \beta \geq H_{n}, \tilde{\beta} \geq \tilde{H}_{n}\right] \\
\leq \frac{\mathrm{e}^{-\langle\theta, z\rangle}}{P_{o, z}\left(l_{1}=0\right)} E_{o} \times E_{z}\left[f(\theta, n, X, \tilde{X}), \beta \geq H_{n}, \tilde{\beta} \geq \tilde{H}_{n}\right] \\
=\frac{1}{P_{o, z}\left(l_{1}=0\right)} E_{o}\left[\exp \left\{\left\langle\theta, X_{H_{n}}\right\rangle-\Lambda_{a}(\theta) H_{n}\right\}, \beta \geq H_{n}\right]^{2} .
\end{gathered}
$$

Here, (3.4) is similar to (3.1). Both facts follow from a standard coupling argument (cf. [2], Proposition 3.7.) Note that

$$
\begin{aligned}
E_{o} & {\left[\exp \left\{\left\langle\theta, X_{H_{n}}\right\rangle-\Lambda_{a}(\theta) H_{n}\right\}, \beta \geq H_{n}\right] } \\
= & \frac{P_{o}(\beta=\infty)}{P_{o}(\beta=\infty)} E_{o}\left[\exp \left\{\left\langle\theta, X_{H_{n}}\right\rangle-\Lambda_{a}(\theta) H_{n}\right\}, \beta \geq H_{n}\right] \\
= & \frac{1}{P_{o}(\beta=\infty)} E_{o}\left[\exp \left\{\left\langle\theta, X_{H_{n}}\right\rangle-\Lambda_{a}(\theta) H_{n}\right\}, \beta \geq H_{n}\right] \\
& \times P_{o}\left(\left\langle X_{i}, e_{1}\right\rangle \geq n \text { for every } i \geq H_{n}\right) \\
= & \frac{1}{P_{o}(\beta=\infty)} E_{o}\left[\exp \left\{\left\langle\theta, X_{H_{n}}\right\rangle-\Lambda_{a}(\theta) H_{n}\right\}, H_{n}=\tau_{k} \text { for some } k \geq 1, \beta=\infty\right] \\
= & \mathbb{E}\left\{g_{n}(\theta, \cdot)\right\} / P_{o}(\beta=\infty) .
\end{aligned}
$$

Therefore, (3.5), Lemma 11 and Lemma 20 imply that

$$
\sup _{n \geq 1} \sup _{z \in \mathbb{V}_{d}^{\prime}} C_{n, z}(\theta) \leq P_{o}(\beta=\infty)^{-2} \sup _{z \in \mathbb{V}_{d}^{\prime}} P_{o, z}\left(l_{1}=0\right)^{-1}\left(\sup _{n \geq 1} \mathbb{E}\left\{g_{n}(\theta, \cdot)\right\}\right)^{2}<\infty
$$


The proof of part (b) of Lemma 21 is more technical. At $\theta=0$,

$$
\begin{aligned}
B(0) & =\sup _{z \in \mathbb{V}_{d}^{\prime}} \sum_{k=1}^{\infty} B_{k, z}(0) \\
& =\sup _{z \in \mathbb{V}_{d}^{\prime}} \sum_{k=1}^{\infty} P_{o, z}\left(k=\inf \left\{l \in \mathcal{L}: l>v_{1}, X_{H_{l}} \neq \tilde{X}_{\tilde{H}_{l}}\right\} \mid l_{1}=0\right) \\
& =\sup _{z \in \mathbb{V}_{d}^{\prime}} P_{o, z}\left(v_{1}<\infty \mid l_{1}=0\right)=1-\inf _{z \in \mathbb{V}_{d}^{\prime}} P_{o, z}\left(v_{1}=\infty \mid l_{1}=0\right)<1
\end{aligned}
$$

by Lemma 20 . For every $\theta \in \mathcal{C}_{a}\left(\kappa_{3}\right)$ and $z \in \mathbb{V}_{d}^{\prime}$,

$$
\begin{aligned}
\sum_{k=1}^{\infty} B_{k, z}(\theta) & =\sum_{k=1}^{\infty} B_{k, z}(0)+\sum_{k=1}^{\infty}\left(B_{k, z}(\theta)-B_{k, z}(0)\right) \quad \text { and } \\
B(\theta) & =\sup _{z \in \mathbb{V}_{d}^{\prime}} \sum_{k=1}^{\infty} B_{k, z}(\theta) \leq \sup _{z \in \mathbb{V}_{d}^{\prime}} \sum_{k=1}^{\infty} B_{k, z}(0)+\sum_{k=1}^{\infty} \sup _{z \in \mathbb{V}_{d}^{\prime}}\left(B_{k, z}(\theta)-B_{k, z}(0)\right) \\
& =B(0)+\sum_{k=1}^{\infty} \sup _{z \in \mathbb{V}_{d}^{\prime}}\left(B_{k, z}(\theta)-B_{k, z}(0)\right)
\end{aligned}
$$

The next three lemmas control the sum in (3.7).

Lemma 24 For every $k \geq 1$ and $\epsilon>0$, there exists $a \kappa_{4}=\kappa_{4}(k, \epsilon) \in\left(0, \kappa_{3}\right)$ such that

$$
\sup _{z \in \mathbb{V}_{d}^{\prime}} \sup _{\theta \in \mathcal{C}_{a}\left(\kappa_{4}\right)}\left(B_{k, z}(\theta)-B_{k, z}(0)\right)<\epsilon
$$

Proof For every $k \geq 1, \theta \in \mathcal{C}_{a}\left(\kappa_{3}\right)$ and $z \in \mathbb{V}_{d}^{\prime}$,

$$
\begin{aligned}
& B_{k, z}(\theta)-B_{k, z}(0)=E_{o, z}\left[\mathrm{e}^{-\langle\theta, z\rangle} f(\theta, k, X, \tilde{X})-1,\right. \\
& \left.k=\inf \left\{l \in \mathcal{L}: l>v_{1}, X_{H_{l}} \neq \tilde{X}_{\tilde{H}_{l}}\right\} \mid l_{1}=0\right] \\
& \leq P_{o, z}\left(l_{1}=0\right)^{-1} E_{o, z}\left[\left(\mathrm{e}^{-\langle\theta, z\rangle} f(\theta, k, X, \tilde{X})-1\right)^{2}\right]^{1 / 2} \\
& \quad \times P_{o, z}\left(k=\inf \left\{l \in \mathcal{L}: l>v_{1}, X_{H_{l}} \neq \tilde{X}_{\tilde{H}_{l}}\right\}\right)^{1 / 2} \\
& \leq P_{o, z}\left(l_{1}=0\right)^{-1} \mathbb{E}\left\{E_{o}^{\omega} \times E_{o}^{T_{z} \omega}\left[(f(\theta, k, X, \tilde{X})-1)^{2}\right]\right\}^{1 / 2} \\
& \quad \times\left(P_{o, z}\left(\sup _{1 \leq i \leq H_{k}}\left|X_{i}\right| \geq \frac{|z|}{2}\right)+P_{o, z}\left(\sup _{1 \leq j \leq \tilde{H}_{k}}\left|\tilde{X}_{j}-z\right| \geq \frac{|z|}{2}\right)\right)^{1 / 2}
\end{aligned}
$$




$$
\begin{aligned}
\leq & P_{o, z}\left(l_{1}=0\right)^{-1}\left(\mathbb{E}\left\{E_{o}^{\omega} \times E_{o}^{T_{z} \omega}\left[f(\theta, k, X, \tilde{X})^{2}\right]\right\}+1\right)^{1 / 2} \\
& \times \sqrt{2} P_{o}\left(\sup _{1 \leq i \leq H_{k}}\left|X_{i}\right| \geq \frac{|z|}{2}\right)^{1 / 2} \\
\leq & P_{o, z}\left(l_{1}=0\right)^{-1}\left(E_{o}\left[\exp \left\{4\left\langle\theta, X_{H_{k}}\right\rangle-4 \Lambda_{a}(\theta) H_{k}\right\}\right]+1\right)^{1 / 2} \\
& \times \sqrt{2} P_{o}\left(\sup _{1 \leq i \leq \tau_{k}}\left|X_{i}\right| \geq \frac{|z|}{2}\right)^{1 / 2} .
\end{aligned}
$$

For every $\epsilon>0$, it follows from (3.9), Corollary 9 and Lemma 20 that there exists an $N \geq 1$ such that

$$
\sup _{\substack{z \in \mathbb{V}_{d}^{\prime} \\|z|>N}} \sup _{\theta \in \mathcal{C}_{a}\left(\kappa_{3} / 4\right)}\left(B_{k, z}(\theta)-B_{k, z}(0)\right)<\epsilon
$$

Note that $\theta \mapsto f(\theta, k, X, \tilde{X})$ is continuous. Hence, for every $k \geq 1$ and $z \in \mathbb{V}_{d}^{\prime}$, the map $\theta \mapsto B_{k, z}(\theta)$ is continuous at 0 by Schwarz's inequality, Corollary 9 and the dominated convergence theorem. Consequently, there exists a $\kappa_{4} \in\left(0, \kappa_{3} / 4\right)$ such that

$$
\sup _{\substack{z \in \mathbb{V}_{d}^{\prime} \\|z| \leq N}} \sup _{\theta \in \mathcal{C}_{a}\left(\kappa_{4}\right)}\left|B_{k, z}(\theta)-B_{k, z}(0)\right|<\epsilon
$$

Clearly, (3.10) and (3.11) imply (3.8).

Lemma 25 There exists a $\kappa_{5} \in\left(0, \kappa_{3}\right)$ such that

$$
\sum_{k=1}^{\infty} \sup _{z \in \mathbb{V}_{d}^{\prime}} \sup _{\theta \in \mathcal{C}_{a}\left(\kappa_{5}\right)} B_{k, z}(\theta)<\infty .
$$

Proof For every $k \geq 1, \kappa \in\left(0, \kappa_{3}\right), \theta \in \mathcal{C}_{a}(\kappa)$ and $z \in \mathbb{V}_{d}^{\prime}$,

$$
\begin{gathered}
B_{k, z}(\theta)=\mathrm{e}^{-\langle\theta, z\rangle} E_{o, z}\left[f(\theta, k, X, \tilde{X}), k=\inf \left\{l \in \mathcal{L}: l>v_{1}, X_{H_{l}} \neq \tilde{X}_{\tilde{H}_{l}}\right\} \mid l_{1}=0\right] \\
=\sum_{j=0}^{k-1} \sum_{z^{\prime} \in \mathbb{V}_{d}} \mathrm{e}^{-\langle\theta, z\rangle} E_{o, z}\left[f(\theta, k, X, \tilde{X}), j=\sup \left\{l \in \mathcal{L}: l \leq \nu_{1}\right\}, \tilde{X}_{\tilde{H}_{j}}-X_{H_{j}}=z^{\prime},\right. \\
\left.k=\inf \left\{l \in \mathcal{L}: l>v_{1}, X_{H_{l}} \neq \tilde{X}_{\tilde{H}_{l}}\right\} \mid l_{1}=0\right] \\
\leq \sum_{j=0}^{k-1} \sum_{z^{\prime} \in \mathbb{V}_{d}} \mathrm{e}^{-\langle\theta, z\rangle} E_{o, z}\left[f(\theta, j, X, \tilde{X}), j \in \mathcal{L}, j \leq v_{1}, \tilde{X}_{\tilde{H}_{j}}-X_{H_{j}}=z^{\prime} \mid l_{1}=0\right]
\end{gathered}
$$




$$
\begin{aligned}
& \quad \times \mathrm{e}^{-\left\langle\theta, z^{\prime}\right\rangle} E_{o, z^{\prime}}[f(\theta, k-j, X, \tilde{X}), \\
& \left.k-j=\inf \left\{l \in \mathcal{L}: l>0, X_{H_{l}} \neq \tilde{X}_{\tilde{H}_{l}}\right\}, k-j>v_{1} \mid l_{1}=0\right] \\
& =\sum_{j=0}^{k-1} \sum_{z^{\prime} \in \mathbb{V}_{d}} \mathrm{e}^{-\langle\theta, z\rangle} E_{o, z}\left[f(\theta, j, X, \tilde{X}), j \in \mathcal{L}, j \leq v_{1}, \tilde{X}_{\tilde{H}_{j}}-X_{H_{j}}=z^{\prime} \mid l_{1}=0\right] h_{k-j, z^{\prime}}(\theta) \\
& \leq \sum_{j=0}^{k-1} \sup _{z^{\prime} \in \mathbb{V}_{d}} \mathrm{e}^{-\langle\theta, z\rangle} E_{O, z}\left[f(\theta, j, X, \tilde{X}), j \in \mathcal{L}, j \leq v_{1}, \tilde{X}_{\tilde{H}_{j}}-X_{H_{j}}=z^{\prime} \mid l_{1}=0\right] \\
& \quad \times \sum_{z^{\prime} \in \mathbb{V}_{d}} h_{k-j, z^{\prime}}(\theta)
\end{aligned}
$$

where, for every $i \geq 1$,

$$
\begin{gathered}
h_{i, z^{\prime}}(\theta):=\mathrm{e}^{-\left\langle\theta, z^{\prime}\right\rangle} E_{o, z^{\prime}}\left[f(\theta, i, X, \tilde{X}), i=\inf \left\{l \in \mathcal{L}: l>0, X_{H_{l}} \neq \tilde{X}_{\tilde{H}_{l}}\right\},\right. \\
\left.i>v_{1} \mid l_{1}=0\right] .
\end{gathered}
$$

For every $z^{\prime} \in \mathbb{V}_{d}$,

$$
\begin{aligned}
& \mathrm{e}^{-\langle\theta, z\rangle} E_{o, z}\left[f(\theta, j, X, \tilde{X}), j \in \mathcal{L}, j \leq v_{1}, \tilde{X}_{\tilde{H}_{j}}-X_{H_{j}}=z^{\prime}, l_{1}=0\right] \\
& \leq \mathrm{e}^{-\langle\theta, z\rangle} E_{o, z}\left[f(\theta, j, X, \tilde{X}),\left\{X_{n}: 0 \leq n<H_{j}\right\} \cap\left\{\tilde{X}_{m}: 0 \leq m<\tilde{H}_{j}\right\}=\emptyset,\right. \\
& \left.\tilde{X}_{\tilde{H}_{j}}-X_{H_{j}}=z^{\prime}, \beta \geq H_{j}, \tilde{\beta} \geq \tilde{H}_{j}\right] \\
& =\mathrm{e}^{-\langle\theta, z\rangle} E_{o} \times E_{z}\left[f(\theta, j, X, \tilde{X}),\left\{X_{n}: 0 \leq n<H_{j}\right\} \cap\left\{\tilde{X}_{m}: 0 \leq m<\tilde{H}_{j}\right\}=\emptyset,\right. \\
& \left.\quad \tilde{X}_{\tilde{H}_{j}}-X_{H_{j}}=z^{\prime}, \beta \geq H_{j}, \tilde{\beta} \geq \tilde{H}_{j}\right] \\
& \leq \mathrm{e}^{-\langle\theta, z\rangle} E_{o} \times E_{z}\left[f(\theta, j, X, \tilde{X}), \tilde{X}_{\tilde{H}_{j}}-X_{H_{j}}=z^{\prime}, \beta \geq H_{j}, \tilde{\beta} \geq \tilde{H}_{j}\right] \\
& =E_{o} \times E_{o}\left[f(\theta, j, X, \tilde{X}), \tilde{X} \tilde{H}_{j}-X_{H_{j}}=z^{\prime}-z, \beta \geq H_{j}, \tilde{\beta} \geq \tilde{H}_{j}\right] \\
& =\frac{P_{o} \times P_{o}(j \in \mathcal{L})}{P_{o} \times P_{o}\left(l_{1}=0\right)} E_{o} \times E_{o}\left[f(\theta, j, X, \tilde{X}), \tilde{X}_{\tilde{H}_{j}}-X_{H_{j}}=z^{\prime}-z, \beta \geq H_{j}, \tilde{\beta} \geq \tilde{H}_{j}\right] \\
& =E_{o} \times E_{o}\left[f(\theta, j, X, \tilde{X}), j \in \mathcal{L}, \tilde{X}_{\tilde{H}_{j}}-X_{H_{j}}=z^{\prime}-z \mid l_{1}=0\right] \\
& =\hat{P}_{o}^{\theta} \times \hat{P}_{o}^{\theta}\left(\exists n, m \text { such that }\left\langle Y_{n}, e_{1}\right\rangle=j \text { and } \tilde{Y}_{m}-Y_{n}=z^{\prime}-z\right)
\end{aligned}
$$

where $\left(Y_{n}\right)_{n \geq 0}$ and $\left(\tilde{Y}_{m}\right)_{m \geq 0}$ denote two independent random walks on $\mathbb{Z}^{d}$, both with transition kernel $q^{\theta}(y)_{y \in \mathbb{Z}^{d}}$ given in (2.5). 
Let $\mu=\mu(\theta):=\hat{E}_{o}^{\theta}\left[\left\langle Y_{1}, e_{1}\right\rangle\right]$. For every $j \geq 1,(3.14)$ is equal to

$$
\begin{aligned}
& \sum_{\left\langle x, e_{1}\right\rangle=j} \hat{P}_{o}^{\theta}\left(\exists n \text { such that } Y_{n}=x\right) \hat{P}_{o}^{\theta}\left(\exists m \text { such that } \tilde{Y}_{m}=x+z^{\prime}-z\right) \\
& \leq \sup _{\left\langle x, e_{1}\right\rangle=j} \hat{P}_{o}^{\theta}\left(\exists n \text { such that } Y_{n}=x\right) \sum_{\left\langle x, e_{1}\right\rangle=j} \hat{P}_{o}^{\theta}\left(\exists m \text { such that } \tilde{Y}_{m}=x+z^{\prime}-z\right) \\
& =\sup _{\left\langle x, e_{1}\right\rangle=j} \sum_{n \geq 1} \hat{P}_{o}^{\theta}\left(Y_{n}=x\right) \hat{P}_{o}^{\theta}\left(\exists m \text { such that }\left\langle\tilde{Y}_{m}, e_{1}\right\rangle=j\right) \\
& \leq \sup _{\left\langle x, e_{1}\right\rangle=j} \sum_{n \geq 1} \hat{P}_{o}^{\theta}\left(Y_{n}=x\right) \\
& =\sup _{\left\langle x, e_{1}\right\rangle=j}\left(\sum_{|n-j / \mu| \leq \sqrt{j / \mu}} \hat{P}_{o}^{\theta}\left(Y_{n}=x\right)+\sum_{|n-j / \mu|>\sqrt{j / \mu}} \hat{P}_{o}^{\theta}\left(Y_{n}=x\right)\right) \\
& \leq S(\theta) j^{-(d-1) / 2 .}
\end{aligned}
$$

Here, (3.15) follows from (2.6) and the local CLT. $S(\theta)$ depends on the mean and covariance of $\left(q^{\theta}(y)\right)_{y \in \mathbb{Z}^{d}}$. In particular, $\sup _{\theta \in \mathcal{C}_{a}\left(\kappa_{3}\right)} S(\theta)<\infty$.

Putting (3.12), (3.14) and (3.15) together, we see that

$$
\begin{aligned}
\sup _{z \in \mathbb{V}_{d}^{\prime}} \sup _{\theta \in \mathcal{C}_{a}(\kappa)} B_{k, z}(\theta) \leq & \frac{\sup _{\theta \in \mathcal{C}_{a}(\kappa)} S(\theta)}{\inf _{z \in \mathbb{V}_{d}^{\prime}} P_{o, z}\left(l_{1}=0\right)} \sum_{j=0}^{k-1} \max (1, j)^{-(d-1) / 2} \\
& \times \sum_{z^{\prime} \in \mathbb{V}_{d}} \sup _{\theta \in \mathcal{C}_{a}(\kappa)} h_{k-j, z^{\prime}}(\theta) \text { and } \\
\sum_{k=1}^{\infty} \sup _{z \in \mathbb{V}_{d}^{\prime}} \sup _{\theta \in \mathcal{C}_{a}(\kappa)} B_{k, z}(\theta) \leq & \frac{\sup _{\theta \in \mathcal{C}_{a}(\kappa)} S(\theta)}{\inf _{z \in \mathbb{V}_{d}^{\prime}} P_{o, z}\left(l_{1}=0\right)}\left(1+\sum_{j=1}^{\infty} j^{-(d-1) / 2}\right) \\
& \times \sum_{i=1}^{\infty} \sum_{z^{\prime} \in \mathbb{V}_{d}} \sup _{\theta \in \mathcal{C}_{a}(\kappa)} h_{i, z^{\prime}}(\theta) .
\end{aligned}
$$

The desired result follows from Lemma 20 and Lemma 26 (stated below.)

Lemma 26 Recall (3.13). There exists a $\kappa_{5} \in\left(0, \kappa_{3}\right)$ such that

$$
\sum_{i=1}^{\infty} \sum_{z \in \mathbb{V}_{d}} \sup _{\theta \in \mathcal{C}_{a}\left(\kappa_{5}\right)} h_{i, z}(\theta)<\infty .
$$

Remark 27 Lemma 26 is proved in Sect. 3.4.

Finally, we are ready to give the 
Proof of part (b) of Lemma 21 Let $\epsilon:=1-B(0)$. Note that $\epsilon>0$ by (3.6). Lemma 25 implies that

$$
\sum_{k=N+1}^{\infty} \sup _{z \in \mathbb{V}_{d}^{\prime}} \sup _{\theta \in \mathcal{C}_{a}\left(\kappa_{5}\right)}\left(B_{k, z}(\theta)-B_{k, z}(0)\right) \leq \sum_{k=N+1}^{\infty} \sup _{z \in \mathbb{V}_{d}^{\prime}} \sup _{\theta \in \mathcal{C}_{a}\left(\kappa_{5}\right)} B_{k, z}(\theta)<\epsilon / 2
$$

for some $\kappa_{5} \in\left(0, \kappa_{3}\right)$ and $N \geq 1$. Also, for every $k \geq 1$, there exists a $\kappa_{4}=$ $\kappa_{4}(k, \epsilon / 2 N) \in\left(0, \kappa_{3}\right)$ such that

$$
\sup _{z \in \mathbb{V}_{d}^{\prime}} \sup _{\theta \in \mathcal{C}_{a}\left(\kappa_{4}\right)}\left(B_{k, z}(\theta)-B_{k, z}(0)\right)<\epsilon / 2 N
$$

by Lemma 24 .

Let $\kappa_{e q}:=\min \left(\kappa_{5}, \kappa_{4}(1, \epsilon / 2 N), \kappa_{4}(2, \epsilon / 2 N), \ldots, \kappa_{4}(N, \epsilon / 2 N)\right)$. Recall (3.7). For every $\theta \in \mathcal{C}_{a}\left(\kappa_{e q}\right)$,

$$
\begin{aligned}
B(\theta) & \leq B(0)+\sum_{k=1}^{\infty} \sup _{z \in \mathbb{V}_{d}^{\prime}}\left(B_{k, z}(\theta)-B_{k, z}(0)\right) \\
& =1-\epsilon+\sum_{k=1}^{N} \sup _{z \in \mathbb{V}_{d}^{\prime}}\left(B_{k, z}(\theta)-B_{k, z}(0)\right)+\sum_{k=N+1}^{\infty} \sup _{z \in \mathbb{V}_{d}^{\prime}}\left(B_{k, z}(\theta)-B_{k, z}(0)\right) \\
& <1-\epsilon+N(\epsilon / 2 N)+\epsilon / 2=1 .
\end{aligned}
$$

\subsection{Tail estimates for joint regenerations}

Recall that our proof of Theorem 5 given in Sect. 2 relies on Lemma 12 which, in turn, is proved in Sect. 3.2 assuming Lemma 21. In Sect. 3.3, the latter assumption is verified using yet another result, namely Lemma 26 . Therefore, in order to complete the proof of Theorem 5, we need to prove Lemma 26.

For every $i \geq 1, \theta \in \mathcal{C}_{a}\left(\kappa_{3}\right)$ and $z \in \mathbb{V}_{d}$, it follows from Hölder's inequality that

$$
\begin{aligned}
h_{i, z}(\theta)= & \frac{\mathrm{e}^{-\langle\theta, z\rangle}}{P_{o, z}\left(l_{1}=0\right)} E_{o, z}\left[f(\theta, i, X, \tilde{X}), i=\inf \left\{l \in \mathcal{L}: l>0, X_{H_{l}} \neq \tilde{X}_{\tilde{H}_{l}}\right\},\right. \\
& \left.\quad i>v_{1}, \beta=\infty, \tilde{\beta}=\infty\right] \\
\leq & E_{o, z}\left[\exp \left\{4\left\langle\theta, X_{H_{i}}\right\rangle-4 \Lambda_{a}(\theta) H_{i}\right\}, \beta=\infty\right]^{1 / 4} \\
& \times \mathrm{e}^{-\langle\theta, z\rangle} E_{o, z}\left[\exp \left\{4\left\langle\theta, \tilde{X}_{\tilde{H}_{i}}\right\rangle-4 \Lambda_{a}(\theta) \tilde{H}_{i}\right\}, \tilde{\beta}=\infty\right]^{1 / 4} \\
& \times P_{o, z}\left(l_{1}=0\right)^{-1} \\
& \times P_{o, z}\left(i=\inf \left\{l \in \mathcal{L}: l>0, X_{H_{l}} \neq \tilde{X}_{\tilde{H}_{l}}\right\}, l_{1}=0\right)^{1 / 4} P_{o, z}\left(i>v_{1}\right)^{1 / 4}
\end{aligned}
$$




$$
\begin{aligned}
= & E_{o}\left[\exp \left\{4\left\langle\theta, X_{H_{i}}\right\rangle-4 \Lambda_{a}(\theta) H_{i}\right\}, \beta=\infty\right]^{1 / 2} \\
& \times P_{o, z}\left(l_{1}=0\right)^{-1} P_{o, z}\left(i=\inf \left\{l \in \mathcal{L}: l>0, X_{H_{l}} \neq \tilde{X}_{\tilde{H}_{l}}\right\}, l_{1}=0\right)^{1 / 4} \\
& \times P_{o, z}\left(i>v_{1}\right)^{1 / 4} .
\end{aligned}
$$

The next four lemmas control the terms in (3.16).

Lemma 28 There exists an $a_{1}<\infty$ such that

$$
E_{o}\left[\exp \left\{4\left\langle\theta, X_{H_{i}}\right\rangle-4 \Lambda_{a}(\theta) H_{i}\right\} \mid \beta=\infty\right] \leq i \mathrm{e}^{a_{1}|\theta| i}
$$

for every $i \geq 1$ and $\theta \in \mathcal{C}_{a}\left(\kappa_{3} / 4\right)$.

Proof Recall $\kappa_{3}$ from Corollary 9.

(a) The non-nestling case: For every $i \geq 1$ and $\theta \in \mathcal{C}_{a}\left(\kappa_{3} / 4\right)$,

$$
\begin{aligned}
& E_{o}\left[\exp \left\{4\left\langle\theta, X_{H_{i}}\right\rangle-4 \Lambda_{a}(\theta) H_{i}\right\} \mid \beta=\infty\right] \\
& \quad \leq E_{o}\left[\exp \left\{\left(4|\theta|+4\left|\Lambda_{a}(\theta)\right|\right) H_{i}\right\} \mid \beta=\infty\right] \\
& \quad \leq E_{o}\left[\exp \left\{8|\theta| \tau_{i}\right\} \mid \beta=\infty\right]=E_{o}\left[\exp \left\{8|\theta| \tau_{1}\right\} \mid \beta=\infty\right]^{i} \\
& \leq E_{o}\left[\exp \left\{2 \kappa_{3} \tau_{1}\right\} \mid \beta=\infty\right]^{4|\theta| i / \kappa_{3}}
\end{aligned}
$$

by Jensen's inequality. Since $a_{1}:=\log E_{o}\left[\exp \left\{2 \kappa_{3} \tau_{1}\right\} \mid \beta=\infty\right]^{4 / \kappa_{3}}<\infty$ by Corollary 9, we are done.

(b) The nestling case: For every $i \geq 1$ and $\theta \in \mathcal{C}_{a}\left(\kappa_{3} / 4\right)$,

$$
\begin{aligned}
& E_{o}\left[\exp \left\{4\left\langle\theta, X_{H_{i}}\right\rangle-4 \Lambda_{a}(\theta) H_{i}\right\} \mid \beta=\infty\right] \leq E_{o}\left[\exp \left\{4|\theta|\left|X_{H_{i}}\right|\right\} \mid \beta=\infty\right] \\
& =\sum_{j=0}^{i-1} \sum_{k=j}^{i-1} E_{o}\left[\exp \left\{4|\theta|\left|X_{H_{i}}\right|\right\}, \tau_{j}<H_{i} \leq \tau_{j+1},\left\langle X_{\tau_{j}}, e_{1}\right\rangle=k \mid \beta=\infty\right] \\
& \leq \sum_{j=0}^{i-1} \sum_{k=j}^{i-1} E_{o}\left[\exp \left\{4|\theta|\left|X_{\tau_{j}}\right|\right\}, \tau_{j}=H_{k} \mid \beta=\infty\right] \\
& \quad \times E_{o}\left[\exp \left\{4|\theta|\left|X_{H_{i-k}}\right|\right\}, H_{i-k} \leq \tau_{1} \mid \beta=\infty\right] \\
& \leq \sum_{j=0}^{i-1} E_{o}\left[\exp \left\{4|\theta|\left|X_{\tau_{j}}\right|\right\} \mid \beta=\infty\right] E_{o}\left[\sup _{1 \leq n \leq \tau_{1}} \exp \left\{4|\theta|\left|X_{n}\right|\right\} \mid \beta=\infty\right] \\
& \leq \sum_{j=0}^{i-1} E_{o}\left[\sup _{1 \leq n \leq \tau_{1}} \exp \left\{4|\theta|\left|X_{n}\right|\right\} \mid \beta=\infty\right]^{j+1}
\end{aligned}
$$




$$
\begin{aligned}
& \leq i E_{O}\left[\sup _{1 \leq n \leq \tau_{1}} \exp \left\{4|\theta|\left|X_{n}\right|\right\} \mid \beta=\infty\right]^{i} . \\
& \leq i E_{O}\left[\sup _{1 \leq n \leq \tau_{1}} \exp \left\{\kappa_{3}\left|X_{n}\right|\right\} \mid \beta=\infty\right]^{4|\theta| i / \kappa_{3}} .
\end{aligned}
$$

Since $a_{1}:=\log E_{o}\left[\sup _{1 \leq n \leq \tau_{1}} \exp \left\{\kappa_{3}\left|X_{n}\right|\right\} \mid \beta=\infty\right]^{4 / \kappa_{3}}<\infty$ by Corollary 9, we are done.

Lemma 29 For every $p \geq 1$, there exists an $A_{1}=A_{1}(p)<\infty$ such that

$$
P_{o, z}\left(i>v_{1}\right) \leq A_{1} i^{p} \max (1,|z|)^{-p}
$$

for every $i \geq 1$ and $z \in \mathbb{V}_{d}$.

Proof For every $i \geq 1, z \in \mathbb{V}_{d}^{\prime}$ and $p \geq 1$,

$$
\begin{aligned}
P_{o, z}\left(i>v_{1}\right) & \leq P_{o, z}\left(\left\{X_{n}: 0 \leq n \leq \tau_{i}\right\} \cap\left\{\tilde{X}_{m}: 0 \leq m \leq \tilde{\tau}_{i}\right\} \neq \emptyset\right) \\
& \leq P_{o, z}\left(\tau_{i} \geq \frac{|z|}{2}\right)+P_{o, z}\left(\tilde{\tau}_{i} \geq \frac{|z|}{2}\right)=2 P_{o}\left(\tau_{i} \geq \frac{|z|}{2}\right) \\
& \leq 2\left(\frac{|z|}{2}\right)^{-p} E_{o}\left[\tau_{i}^{p}\right]=2^{p+1}|z|^{-p} E_{o}\left[\left\{\tau_{1}+\cdots+\left(\tau_{i}-\tau_{i-1}\right)\right\}^{p}\right] \\
& \leq 2^{p+1}|z|^{-p} i^{p-1} E_{o}\left[\tau_{1}^{p}+\cdots+\left(\tau_{i}-\tau_{i-1}\right)^{p}\right] \\
& =2^{p+1}|z|^{-p} i^{p-1}\left(E_{o}\left[\tau_{1}^{p}\right]+(i-1) E_{o}\left[\tau_{1}^{p} \mid \beta=\infty\right]\right) \\
& \leq 2^{p+1} P_{o}(\beta=\infty)^{-1} E_{o}\left[\tau_{1}^{p}\right] i^{p}|z|^{-p}
\end{aligned}
$$

by Hölder's inequality. Since $A_{1}(p):=2^{p+1} P_{o}(\beta=\infty)^{-1} E_{o}\left[\tau_{1}^{p}\right]<\infty$ by Corollary 9 , we are done.

Lemma $30 \sup _{z \in \mathbb{V}_{d}} E_{o, z}\left[\mathrm{e}^{a_{3} l^{+}} \mid l_{1}=0\right]<\infty$ for some $a_{3}>0$, where $l^{+}:=\inf \{l \in$ $\mathcal{L}: l>0\}$.

Proof For every nearest-neighbor path $\left(x_{i}\right)_{i \geq 0}$ on $\mathbb{Z}^{d}$, define

$$
\begin{aligned}
& \beta^{\prime}\left(\left(x_{i}\right)_{i \geq 0}\right):=\inf \left\{i \geq 1:\left\langle x_{i}, e_{1}\right\rangle<\left\langle x_{o}, e_{1}\right\rangle\right\} \text { and } \\
& M\left(\left(x_{i}\right)_{i \geq 0}\right):=\sup \left\{\left\langle x_{i}, e_{1}\right\rangle: 0 \leq i<\beta^{\prime}\left(\left(x_{i}\right)_{i \geq 0}\right)\right\} .
\end{aligned}
$$

In particular, $\beta^{\prime}(X)=\beta$ and $\beta^{\prime}(\tilde{X})=\tilde{\beta}$ for $X=\left(X_{n}\right)_{n \geq 0}$ and $\tilde{X}=\left(\tilde{X}_{m}\right)_{m \geq 0}$. Define

$$
\begin{aligned}
\lambda & =\lambda(X, \tilde{X}):=(M(X) \wedge M(\tilde{X}))+1, \quad \lambda_{1}:=1 \text { and } \\
\lambda_{j+1} & :=\lambda\left(\left(X_{n}\right)_{n \geq H_{\lambda_{j}}},\left(\tilde{X}_{m}\right)_{m \geq \tilde{H}_{\lambda_{j}}}\right)
\end{aligned}
$$


for every $j \geq 1$. It is easy to see that $l^{+}:=\sup \left\{\lambda_{j}: \lambda_{j}<\infty\right\}$ when $X_{o} \in \mathbb{V}_{d}$ and $\tilde{X}_{o} \in \mathbb{V}_{d}$.

For every $z \in \mathbb{V}_{d}$,

$$
\begin{aligned}
E_{o, z}\left[\mathrm{e}^{\kappa_{3} \lambda}, \lambda<\infty\right] & \leq E_{o, z}\left[\mathrm{e}^{\kappa_{3}(M(X)+1)}, \beta<\infty\right]+E_{o, z}\left[\mathrm{e}^{\kappa_{3}(M(\tilde{X})+1)}, \tilde{\beta}<\infty\right] \\
& =2 E_{o}\left[\mathrm{e}^{\kappa_{3}(M(X)+1)}, \beta<\infty\right] \leq 2 E_{o}\left[\sup _{1 \leq n \leq \tau_{1}} \mathrm{e}^{\kappa_{3}\left|X_{n}\right|}, \beta<\infty\right]
\end{aligned}
$$

By Hölder's inequality,

$$
\begin{aligned}
E_{o, z}\left[\mathrm{e}^{a \lambda}, \lambda<\infty\right] & \leq E_{o, z}\left[\mathrm{e}^{\kappa_{3} \lambda}, \lambda<\infty\right]^{a / \kappa_{3}} P_{o, z}(\lambda<\infty)^{1-a / \kappa_{3}} \\
& \leq\left(2 E_{o}\left[\sup _{1 \leq n \leq \tau_{1}} \mathrm{e}^{\kappa_{3}\left|X_{n}\right|}\right]\right)^{a / \kappa_{3}}\left(1-P_{o, z}\left(l_{1}=0\right)\right)^{1-a / \kappa_{3}}
\end{aligned}
$$

for every $a \in\left(0, \kappa_{3}\right)$. Therefore, it follows from Corollary 9 and Lemma 20 that

$$
\begin{aligned}
\sup _{z \in \mathbb{V}_{d}} E_{o, z}\left[\mathrm{e}^{a_{3} \lambda}, \lambda<\infty\right] \leq & \left(2 E_{o}\left[\sup _{1 \leq n \leq \tau_{1}} \mathrm{e}^{\kappa_{3}\left|X_{n}\right|}\right]\right)^{a_{3} / \kappa_{3}} \\
& \times\left(1-\inf _{z \in \mathbb{V}_{d}} P_{o, z}\left(l_{1}=0\right)\right)^{1-a_{3} / \kappa_{3}}<1
\end{aligned}
$$

for some $a_{3} \in\left(0, \kappa_{3}\right)$.

For every $j \geq 2$,

$$
\begin{aligned}
E_{o, z}\left[\mathrm{e}^{a_{3} \lambda_{j}}, \lambda_{j}<\infty\right]= & \sum_{z^{\prime} \in \mathbb{V}_{d}} E_{o, z}\left[\mathrm{e}^{a_{3} \lambda_{j}}, \tilde{X}_{\tilde{H}_{\lambda_{j-1}}}-X_{H_{\lambda_{j-1}}}=z^{\prime}, \lambda_{j}<\infty\right] \\
= & \sum_{z^{\prime} \in \mathbb{V}_{d}} E_{o, z}\left[\mathrm{e}^{a_{3} \lambda_{j-1}}, \lambda_{j-1}<\infty, \tilde{X}_{\tilde{H}_{\lambda_{j-1}}}-X_{H_{\lambda_{j-1}}}=z^{\prime}\right] \\
& \times E_{o, z^{\prime}}\left[\mathrm{e}^{a_{3} \lambda}, \lambda<\infty\right] \\
\leq & E_{o, z}\left[\mathrm{e}^{a_{3} \lambda_{j-1}}, \lambda_{j-1}<\infty\right] \sup _{z^{\prime} \in \mathbb{V}_{d}} E_{o, z^{\prime}}\left[\mathrm{e}^{a_{3} \lambda}, \lambda<\infty\right] .
\end{aligned}
$$

By induction,

$$
\sup _{z \in \mathbb{V}_{d}} E_{o, z}\left[\mathrm{e}^{a_{3} \lambda_{j}}, \lambda_{j}<\infty\right] \leq \mathrm{e}^{a_{3}}\left(\sup _{z \in \mathbb{V}_{d}} E_{o, z}\left[\mathrm{e}^{a_{3} \lambda}, \lambda<\infty\right]\right)^{j-1} .
$$


Therefore,

$$
\begin{aligned}
\sup _{z \in \mathbb{V}_{d}} E_{o, z}\left[\mathrm{e}^{a_{3} l^{+}}\right] & \leq \sum_{j=1}^{\infty} \sup _{z \in \mathbb{V}_{d}} E_{o, z}\left[\mathrm{e}^{a_{3} l^{+}}, l^{+}=\lambda_{j}\right] \leq \sum_{j=1}^{\infty} \sup _{z \in \mathbb{V}_{d}} E_{o, z}\left[\mathrm{e}^{a_{3} \lambda_{j}}, \lambda_{j}<\infty\right] \\
& \leq \sum_{j=1}^{\infty} \mathrm{e}^{a_{3}}\left(\sup _{z \in \mathbb{V}_{d}} E_{o, z}\left[\mathrm{e}^{a_{3} \lambda}, \lambda<\infty\right]\right)^{j-1} \\
& =\mathrm{e}^{a_{3}}\left(1-\sup _{z \in \mathbb{V}_{d}} E_{o, z}\left[\mathrm{e}^{a_{3} \lambda}, \lambda<\infty\right]\right)^{-1}<\infty
\end{aligned}
$$

by (3.17). This implies the desired result since $\inf _{z \in \mathbb{V}_{d}} P_{o, z}\left(l_{1}=0\right)>0$ by Lemma 20 .

Lemma 31 There exist $a_{2}>0$ and $A_{2}<\infty$ such that $P_{o, z}\left(l^{*}=i \mid l_{1}=0\right) \leq$ $A_{2} \mathrm{e}^{-a_{2} i}$ for every $i \geq 1$ and $z \in \mathbb{V}_{d}$, where $l^{*}:=\inf \left\{l \in \mathcal{L}: l>0, X_{H_{l}} \neq \tilde{X}_{\tilde{H}_{l}}\right\}$.

Proof Fix $a_{3}$ as in Lemma 30. Define $v_{1}^{+}:=\inf \left\{m>0: X_{i}=\tilde{X}_{j}\right.$ for some $i \geq 0, j \geq 0$, and $\left.\left\langle X_{i}, e_{1}\right\rangle=m\right\}$. For every $z \in \mathbb{V}_{d}$ and $a \in\left(0, a_{3}\right)$, by Hölder's inequality,

$$
\begin{aligned}
& E_{o, z}\left[\mathrm{e}^{a l^{+}}, v_{1}^{+}<\infty \mid l_{1}=0\right] \leq E_{o, z}\left[\mathrm{e}^{a_{3} l^{+}} \mid l_{1}=0\right]^{a / a_{3}} P_{o, z}\left(v_{1}^{+}<\infty \mid l_{1}=0\right)^{1-a / a_{3}} \text { and } \\
& \sup _{z \in \mathbb{V}_{d}} E_{o, z}\left[\mathrm{e}^{a l^{+}}, v_{1}^{+}<\infty \mid l_{1}=0\right] \leq\left(\sup _{z \in \mathbb{V}_{d}} E_{o, z}\left[\mathrm{e}^{a_{3} l^{+}} \mid l_{1}=0\right]\right)^{a / a_{3}} \\
& \times\left(\sup _{z \in \mathbb{V}_{d}} P_{o, z}\left(v_{1}^{+}<\infty \mid l_{1}=0\right)\right)^{1-a / a_{3}} .
\end{aligned}
$$

It is an easy consequence of (1.1) and Lemma 20 that $\sup _{z \in \mathbb{V}_{d}} P_{o, z}\left(v_{1}^{+}<\infty \mid l_{1}=0\right)<1$. Hence,

$$
\sup _{z \in \mathbb{V}_{d}} E_{o, z}\left[\mathrm{e}^{a_{2} l^{+}}, v_{1}^{+}<\infty \mid l_{1}=0\right]<1
$$

for some $a_{2} \in\left(0, a_{3}\right)$. 
It follows from the regeneration structure that

$$
\begin{aligned}
E_{o, z}\left[\mathrm{e}^{a_{2} l^{*}} \mid l_{1}=0\right]= & E_{o, z}\left[\mathrm{e}^{a_{2} l^{*}}, l^{+}<l^{*} \mid l_{1}=0\right]+E_{o, z}\left[\mathrm{e}^{a_{2} l^{*}}, l^{+}=l^{*} \mid l_{1}=0\right] \\
= & E_{o, z}\left[\mathrm{e}^{a_{2} l^{+}}, X_{H_{l+}}=\tilde{X}_{\tilde{H}_{l^{+}}} \mid l_{1}=0\right] E_{o, o}\left[\mathrm{e}^{a_{2} l^{*}} \mid l_{1}=0\right] \\
& +E_{o, z}\left[\mathrm{e}^{a_{2} l^{+}}, l^{+}=l^{*} \mid l_{1}=0\right] \\
\leq & E_{o, z}\left[\mathrm{e}^{a_{2} l^{+}}, v_{1}^{+}<\infty \mid l_{1}=0\right] E_{o, o}\left[\mathrm{e}^{a_{2} l^{*}} \mid l_{1}=0\right] \\
& +E_{o, z}\left[\mathrm{e}^{a_{2} l^{+}} \mid l_{1}=0\right] .
\end{aligned}
$$

Therefore,

$$
\begin{aligned}
\sup _{z \in \mathbb{V}_{d}} E_{o, z}\left[\mathrm{e}^{a_{2} l^{*}} \mid l_{1}=0\right] \leq & \sup _{z \in \mathbb{V}_{d}} E_{o, z}\left[\mathrm{e}^{a_{2} l^{+}}, v_{1}^{+}<\infty \mid l_{1}=0\right] \\
& \times \sup _{z \in \mathbb{V}_{d}} E_{o, z}\left[\mathrm{e}^{a_{2} l^{*}} \mid l_{1}=0\right]+\sup _{z \in \mathbb{V}_{d}} E_{o, z}\left[\mathrm{e}^{a_{2} l^{+}} \mid l_{1}=0\right]
\end{aligned}
$$

and

$$
\begin{aligned}
A_{2}:=\sup _{z \in \mathbb{V}_{d}} E_{o, z}\left[\mathrm{e}^{a_{2} l^{*}} \mid l_{1}=0\right] \leq & \left(1-\sup _{z \in \mathbb{V}_{d}} E_{o, z}\left[\mathrm{e}^{a_{2} l^{+}}, v_{1}^{+}<\infty \mid l_{1}=0\right]\right)^{-1} \\
& \times \sup _{z \in \mathbb{V}_{d}} E_{o, z}\left[\mathrm{e}^{a_{2} l^{+}} \mid l_{1}=0\right]<\infty
\end{aligned}
$$

Finally, for every $i \geq 1$ and $z \in \mathbb{V}_{d}$,

$$
P_{o, z}\left(l^{*}=i \mid l_{1}=0\right) \leq E_{o, z}\left[\mathrm{e}^{a_{2} l^{*}} \mid l_{1}=0\right] \mathrm{e}^{-a_{2} i} \leq A_{2} \mathrm{e}^{-a_{2} i}
$$

by Chebyshev's inequality.

Proof of Lemma 26 Recall (3.16). For every $\kappa_{5} \in\left(0, \kappa_{3} / 4\right)$ and $p \geq 1$, Lemmas 28, 29 and 31 imply that

$$
\begin{aligned}
& \sum_{i=1}^{\infty} \sum_{z \in \mathbb{V}_{d}} \sup _{\theta \in \mathcal{C}_{a}\left(\kappa_{5}\right)} h_{i, z}(\theta) \\
& \leq \sum_{i=1}^{\infty} \sum_{z \in \mathbb{V}_{d}} \sup _{\theta \in \mathcal{C}_{a}\left(\kappa_{5}\right)} E_{o}\left[\exp \left\{4\left\langle\theta, X_{H_{i}}\right\rangle-4 \Lambda_{a}(\theta) H_{i}\right\}, \beta=\infty\right]^{1 / 2} \\
& \quad \times P_{o, z}\left(l_{1}=0\right)^{-1} \\
& \quad \times P_{o, z}\left(i=\inf \left\{l \in \mathcal{L}: l>0, X_{H_{l}} \neq \tilde{X}_{\tilde{H}_{l}}\right\}, l_{1}=0\right)^{1 / 4} \\
& \quad \times P_{o, z}\left(i>v_{1}\right)^{1 / 4}
\end{aligned}
$$




$$
\begin{aligned}
\leq & \sum_{i=1}^{\infty} \sum_{z \in \mathbb{V}_{d}} \sup _{\theta \in \mathcal{C}_{a}\left(\kappa_{5}\right)}\left(i \mathrm{e}^{a_{1}|\theta| i}\right)^{1 / 2} P_{o, z}\left(l_{1}=0\right)^{-1}\left(A_{2} \mathrm{e}^{-a_{2} i}\right)^{1 / 4} \\
& \times\left(A_{1} i^{p} \max (1,|z|)^{-p}\right)^{1 / 4} \\
\leq & \frac{\left(A_{1} A_{2}\right)^{1 / 4}}{\inf _{z \in \mathbb{V}_{d}} P_{o, z}\left(l_{1}=0\right)}\left(\sum_{i=1}^{\infty} i^{1 / 2+p / 4} \exp \left\{\left(2 a_{1} \kappa_{5}-a_{2}\right) i / 4\right\}\right) \\
& \times\left(1+\sum_{z \in \mathbb{V}_{d}^{\prime}}|z|^{-p / 4}\right)
\end{aligned}
$$

for some $a_{1}<\infty, a_{2}>0, A_{1}<\infty$ and $A_{2}<\infty$. Clearly, (3.18) is finite when $p>4 d$ and $\kappa_{5} \in\left(0, a_{2} / 2 a_{1}\right)$.

Acknowledgments I sincerely thank O. Zeitouni for many valuable discussions and comments.

Open Access This article is distributed under the terms of the Creative Commons Attribution Noncommercial License which permits any noncommercial use, distribution, and reproduction in any medium, provided the original author(s) and source are credited.

\section{References}

1. Aidékon, E.: Large deviations for random walks in random environment on a Galton-Watson tree. (2008). arXiv:0811.0438

2. Berger, N., Zeitouni, O.: A quenched invariance principle for certain ballistic random walks in i.i.d. environments. In: In and Out of Equilibrium. 2, volume 60 of Progr. Probab., pp. 137-160. Birkhäuser, Basel (2008)

3. Breiman, L.: Probability, volume 7 of Classics in Applied Mathematics. Society for Industrial and Applied Mathematics (SIAM), Philadelphia, 1992. Corrected reprint of the 1968 original

4. Comets, F., Gantert, N., Zeitouni, O.: Quenched, annealed and functional large deviations for onedimensional random walk in random environment. Probab. Theory Relat. Fields 118(1), 65-114 (2000)

5. Comets, F., Shiga, T., Yoshida, N.: Directed polymers in a random environment: path localization and strong disorder. Bernoulli 9(4), 705-723 (2003)

6. Dembo, A., Gantert, N., Peres, Y., Zeitouni, O.: Large deviations for random walks on Galton-Watson trees: averaging and uncertainty. Probab. Theory Relat. Fields 122(2), 241-288 (2002)

7. Dembo, A., Gantert, N., Zeitouni, O.: Large deviations for random walk in random environment with holding times. Ann. Probab. 32(1B), 996-1029 (2004)

8. Dembo, A., Zeitouni, O.: Large Deviations Techniques and Applications, volume 38 of Applications of Mathematics, 2nd edn. Springer-Verlag, New York (1998)

9. Flury, M.: Coincidence of Lyapunov exponents for random walks in weak random potentials. Ann. Probab. 36(4), 1528-1583 (2008)

10. Greven, A., den Hollander, F.: Large deviations for a random walk in random environment. Ann. Probab. 22(3), 1381-1428 (1994)

11. Krantz, S.G., Parks, H.R.: The implicit function theorem. In: History, Theory, and Applications. Birkhäuser, Boston (2002)

12. Peterson, J., Zeitouni, O.: On the annealed large deviation rate function for a multi-dimensional random walk in random environment. ALEA (2008, to appear). arXiv:0812.3619

13. Rassoul-Agha, F.: Large deviations for random walks in a mixing random environment and other (non-Markov) random walks. Comm. Pure Appl. Math. 57(9), 1178-1196 (2004)

14. Rassoul-Agha, F., Seppäläinen, T.: Almost sure functional central limit theorem for ballistic random walk in random environment. Ann. Inst. Henri Poincaré Probab. Stat. 45(2), 373-420 (2009) 
15. Tyrrell Rockafellar, R.: Convex Analysis. Princeton Landmarks in Mathematics. Princeton University Press, Princeton (1997). Reprint of the 1970 original, Princeton Paperbacks

16. Rosenbluth, J.: Quenched large deviations for multidimensional random walk in random environment: a variational formula. PhD thesis in Mathematics, New York University (2006). arXiv:0804.1444

17. Sznitman, A.-S.: Slowdown estimates and central limit theorem for random walks in random environment. J. Eur. Math. Soc. 2(2), 93-143 (2000)

18. Sznitman, A.-S.: On a class of transient random walks in random environment. Ann. Probab. 29(2), 724765 (2001)

19. Sznitman, A.-S., Zerner, M.: A law of large numbers for random walks in random environment. Ann. Probab. 27(4), 1851-1869 (1999)

20. Varadhan, S.R.S.: Large deviations for random walks in a random environment. Comm. Pure Appl. Math. 56(8), 1222-1245 (2003). Dedicated to the memory of Jürgen K. Moser

21. Yilmaz, A.: Averaged large deviations for random walk in a random environment. Ann. Inst. H. Poincaré Probab. Statist. (2008, to appear). arXiv:0809.3467

22. Yilmaz, A.: Large deviations for random walk in a space-time product environment. Ann. Probab. 37(1), 189-205 (2009)

23. Yilmaz, A.: Quenched large deviations for random walk in a random environment. Comm. Pure Appl. Math. 62(8), 1033-1075 (2009)

24. Zeitouni, O.: Random walks in random environments. J. Phys. A 39(40), R433-R464 (2006)

25. Zerner, M.P.W.: Lyapounov exponents and quenched large deviations for multidimensional random walk in random environment. Ann. Probab. 26(4), 1446-1476 (1998)

26. Zygouras, N.: Lyapounov norms for random walks in low disorder and dimension greater than three. Probab. Theory Relat. Fields 143(3-4), 615-642 (2009) 